\title{
Multiscale simulation of molecular gas flows by the general synthetic iterative scheme
}

\author{
Wei Su ${ }^{\mathrm{a}, 1}$, Yonghao Zhang ${ }^{\mathrm{b}}$, Lei $\mathrm{Wu}^{\mathrm{c}, *}$ \\ a James Weir Fluids Laboratory, Department of Mechanical and Aerospace Engineering, University of Strathclyde, Glasgow \\ G1 1 XJ, UK \\ ${ }^{b}$ School of Engineering, The University of Edinburgh, Edinburgh EH9 3FB, UK \\ ${ }^{c}$ Department of Mechanics and Aerospace Engineering, Southern University of Science and Technology, Shenzhen 518055, \\ China
}

\begin{abstract}
The in-depth knowledge of rarefied gas dynamics is crucial to address challenges in a wide range of engineering problems, where the gas flow is usually multiscale, i.e. covering a wide range of Knudsen numbers. As the traditional Navier-Stokes equations fail, gas kinetic equations are required to model the flow. So far, very few numerical methods are designed to efficiently solve the multiscale gas dynamics and reveal the role of internal degrees of freedom of gas molecules. In this work, a general synthetic iterative scheme (GSIS) is proposed to find steady-state solution of the gas kinetic equations for molecular gas flows accurately and efficiently, where the gas kinetic equations are solved together with the macroscopic synthetic equations that expedite the solution towards the steady state. In the macroscopic synthetic equations, while the momentum equation is the same as that used in the GSIS for monatomic gas, two energy equations are introduced here for polyatomic gases: one is for the translational energy and the other for internal energy; these equations are derived exactly from the gas kinetic equations hence no approximation is made in the final solution. The Fourier stability analysis is performed to show that the GSIS permits fast convergence to steady-state solutions in the entire flow regime; meanwhile the asymptotic analysis shows that the GSIS recovers the Navier-Stokes equations when the Knudsen number is small, even on the spatial grid with cell size much larger than the molecular mean free path. With all these unique features, several challenging numerical examples are given to show that the proposed GSIS is a promising tool to simulate multiscale molecular gas flows and investigate the effects of internal degrees of freedom.
\end{abstract}

Keywords: rarefied gas flow, general synthetic iterative scheme, molecular gas, fast convergence, asymptotic Navier-Stokes preserving

\section{Introduction}

In-depth knowledge of the rarefied gas dynamics is crucial to address scientific and engineering challenges in a wide range of applications, such as high-altitude space vehicles, micro-electromechanical systems, unconventional gas production, and vacuum science. For instance, in space exploration, understanding the physico-chemical phenomena that dictate the flow around re-entry capsules allows optimal design of aerodynamic shape and heat shield to fulfill the payload and cost budget, key to success of ambitious space missions. In realistic circumstances, the dilute gas flows are usually multiscale in time and/or space, which span a wide range of Knudsen numbers $(K n$, defined as the ratio of the molecular mean free path to a characteristic flow length, or the ratio of a characteristic flow frequency to the molecular mean collision

\footnotetext{
${ }^{*}$ Corresponding author

Email address: wul@sustech.edu.cn (Lei Wu)

${ }^{1}$ Now at School of Engineering, The University of Edinburgh, Edinburgh EH9 3FB, UK
} 

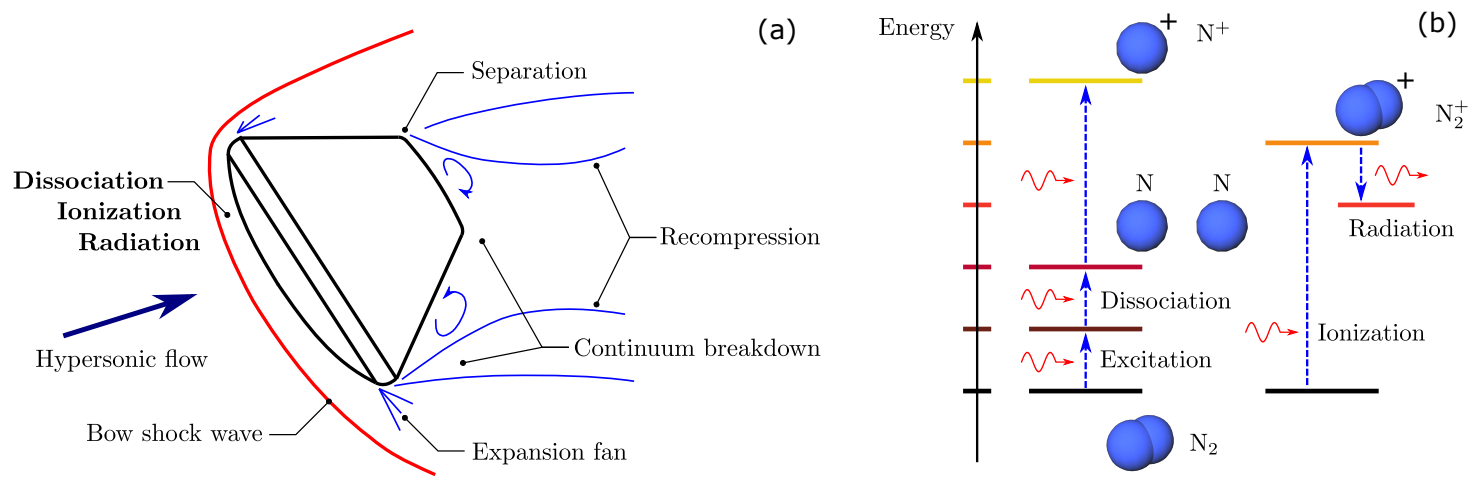

Figure 1: (a) Schematic of flow physics around a re-entry capsule. (b) Schematic diagram of internal excitation, dissociation, ionization and radiation of the nitrogen species.

frequency). Figure 1(a) illustrates the complexity of flow physics around a re-entry capsule, where the space vehicle moves with considerable velocity varying from $7.8 \mathrm{~km} / \mathrm{s}$ when returning from the International Space Station to more than $12 \mathrm{~km} / \mathrm{s}$ for interplanetary travels. Due to the acute interaction with the atmosphere, a strong bow shock wave is generated in front of the capsule; meanwhile supersonic expansion, flow separation, re-circulation, and re-compression take place as the flow passes through it. Behind the shock wave, a massive amount of the kinetic energy of free stream is converted into the internal energy of surrounding gas, which results in intense convective and radiative heating to the space vehicle. The intermolecular collisions also promote energy exchanges between the translational and internal (i.e. rotational, vibrational and electronic) modes; and eventually leads to nonequilibrium chemical reactions including dissociation and ionization, where some species of the products are strong radiators, taken the nitrogen as an example in Figure 1(b). Depending on the flight altitude, atmospheric re-entry usually occurs in the continuum/nearcontinuum flow regimes [1, e.g. at $100 \mathrm{~km}$ for Earth, however the continuum-fluid hypothesis breaks down in the near wake of space vehicle and the kinetic theory is needed to describe the rarefied gas dynamics. Although considerable progress has been accomplished, there remain large uncertainties associated with the approaches to depict the nonequilibrium dynamics of molecular gas flows with such complexities.

Continuing efforts are required to develop accurate physical models and computationally efficient methods for multiscale flow simulations. Benefited from the technological advances in high-performance computing, better understanding of rarefied gas dynamics has been achieved through solving the Boltzmann equation in the framework of gas kinetic theory [2, 3. In particular, the direct simulation Monte Carlo (DSMC) method 4, which is equivalent to the solution of Boltzmann equation for monatomic gas [5], has achieved overwhelming success in simulating hypersonic flows due to its unconditional stability. To enable DSMC to simulate low-speed flows, the information-preserved and low-variance DSMC methods were developed [6] 7 . However, DSMC becomes prohibitively expensive as the gas flow approaches the near-continuum regime. This is because the streaming and collision of gas molecules in DSMC are decoupled on the length and time scales comparable to the cell size of spatial grid and simulation time step which, in order to suppress the numerical dissipation, are required to be smaller than the molecular mean free path and the mean collision time 8, respectively. Moreover, DSMC has explicit time marching, where the time step is restricted by the physical collision time such that all the computations are unsteady and the steady solution is obtained as the long time asymptotic state of the transient flow [9]. Since the information (e.g. perturbation in the flow field) propagation relies on molecular streaming, the number of time steps to reach steady state becomes extremely large when $K n$ is small.

On the other hand, phenomenological models are introduced in DSMC to describe the intermolecular collisions and internal relaxation of molecular gas. Some principles need to be satisfied to guarantee accuracy, one of which is that the experimental values of transport coefficients should be recovered. For monatomic gases, the variable-hard-sphere and variable-soft-sphere models [4, 10, can produce correct values of shear viscosity, thermal conductivity and self-diffusion coefficient. When considering molecular gas flow, additional 
transport coefficients including the bulk viscosity as well as the translational, rotational and vibrational thermal conductivities arise due to energy exchanges between the translational and internal modes. The widely used Borgnakke \& Larsen phenomenological model [11] is able to recover the energy exchange rates so that the bulk viscosity can be correctly obtained, but it has no mechanism to reproduce the total thermal conductivity and its components [12. These physical parameters, however, affect the dynamics of molecular gas flows under nonequilibrium conditions (e.g. shock wave structure [13, line shape of the Rayleigh-Brillouin scattering 12 and thermal transpiration in micro devices) if the translational thermal conductivity is not correct, and even under equilibrium conditions if the total thermal conductivity is not correct.

The gas kinetic equations can be alternatively solved by deterministic approaches [14, 15, 16. When the Boltzmann equation is extended to the Wang-Chang \& Uhlenbeck equation [17] for molecular gases, additional internal degrees of freedom yields a collision operator that is much more complicated than the Boltzmann collision operator for monatomic gas. In practice, gas kinetic models are introduced to reduce the computational complexity in the Wang-Chang \& Uhlenbeck equation [18, 19, 20, 21, 22, 23, some of them are able to recover all the transport coefficients. Conventional techniques of computational fluid dynamics are then utilized to directly discretize the governing equation over a computational grid. Although highorder and implicit schemes would be preferred, most of the solvers treat the movement and collision of gas molecules in a decoupled manner just as that in DSMC. As a consequence, the restriction on spatial cell size and computational time step still exists [24. Meanwhile, information is exchanged through evolution of gas molecular system, thus the conventional iterative scheme (CIS) used by the deterministic solvers for finding steady-state solutions also converges extremely slowly when $K n$ is small [25, 26].

Inspired by the fast iterative methods originally devised to accelerate the simulation of radiation transport problems [27, 28, the present authors have developed a general synthetic iterative scheme (GSIS) to tackle the difficulties of CIS for general rarefied gas dynamics rather than specific problems [25, 229, 30, 31, 32, such that steady-state solutions are obtained within dozens of iterations at any Knudsen number [33, 34]. The key ingredient of GSIS is that macroscopic synthetic equations are simultaneously solved with the gas kinetic equation, from which the macroscopic flow properties are obtained to guide the evolution of gas molecular system thus achieving fast convergence. Moreover, the GSIS asymptotically preserves the NavierStokes equations at the continuum limit 35, so that the restriction on spatial grid cell size and/or time step is eliminated. These advantages make the GSIS a promising tool for simulation of multiscale rarefied gas flows [36].

In this work, we will further explore the GSIS in simulating molecular gas flows, where energy exchanges between the translation and internal modes bring new flow physics and pose difficulties to formulate macroscopic synthetic equations. We will establish the GSIS based on the kinetic model developed by Wu et al. for molecular gases considering rotational degrees of freedom [37. This model has the following advantages: 1) it is reduced to the Boltzmann equation for monatomic gas when the exchange of translational and rotational energies is absent, so that the effect of intermolecular potential and the shear viscosity can be considered; 2) transport coefficients including the bulk viscosity, the translational and rotational thermal conductivities can be freely adjusted to the experimental measured values; 3) although from Figure 1 we see that there are many internal modes, we believe the understanding of rotational mode is the first crucial step to study the rarefied molecular gas flows. Also, the methodology can be straightforwardly applied to kinetic models with more internal modes 22,23 . In the following sections we will only present the extended GSIS for linearized problems, as these problems permit the linear Fourier stability analysis, hence we will know the property of GSIS in details. If the method works for linear problems, according to our recent work 34, it should also work for nonlinear problems.

The remainder of the paper is organized as follows. The kinetic model for molecular gas and the CIS are given in Section 2, while the formulation and procedure of GSIS for molecular gas are presented in Section 3 In Section 4, we rigorously analyze the fast convergence and asymptotic preserving of GSIS by conducting the Fourier stability analysis and the Chapman-Enskog expansion. Five different thermal driven flows are simulated in Section 5 to demonstrate performance of the proposed method. Section 6 concludes the paper. 


\section{Gas kinetic equations and conventional iterative scheme}

For simplicity, we consider a molecular gas with three translational and $d_{r}$ rotational degrees of freedom, while its vibrational energy is not excited. In gas kinetic theory, the dilute gas system is described by the distribution function $f(t, \boldsymbol{x}, \boldsymbol{v}, I)$, where $t$ is the time, $\boldsymbol{x}=\left(x_{1}, x_{2}, x_{3}\right)$ is the Cartesian coordinate, $\boldsymbol{v}=\left(v_{1}, v_{2}, v_{3}\right)$ is the translational velocity of gas molecules, and $I$ is the rotational energy [37]. In order to improve computational efficiency, two reduced velocity distribution functions $G(t, \boldsymbol{x}, \boldsymbol{v})=\int_{0}^{\infty} f(t, \boldsymbol{x}, \boldsymbol{v}, I) \mathrm{d} I$ and $R(t, \boldsymbol{x}, \boldsymbol{v})=\int_{0}^{\infty} f(t, \boldsymbol{x}, \boldsymbol{v}, I) I^{2 / d_{r}} \mathrm{~d} I$ are introduced to eliminate the dependence on $I$. We further assume the gas flow is driven by sufficiently small perturbation, hence the reduced velocity distribution functions deviate slightly from the global equilibrium, which can be linearized as

$$
\begin{array}{r}
G(t, \boldsymbol{x}, \boldsymbol{v})=F_{e q}(\boldsymbol{v})+h_{0}(t, \boldsymbol{x}, \boldsymbol{v}), \\
R(t, \boldsymbol{x}, \boldsymbol{v})=\frac{d_{r}}{2} F_{e q}(\boldsymbol{v})+h_{1}(t, \boldsymbol{x}, \boldsymbol{v}),
\end{array}
$$

where the equilibrium distribution function is given by

$$
F_{e q}=\pi^{-3 / 2} \exp \left(-|\boldsymbol{v}|^{2}\right)
$$

and the small perturbations $h_{0}, h_{1}$ satisfy $\left|h_{0} / F_{e q}\right| \ll 1$ and $\left|h_{1} / F_{e q}\right| \ll 1$. On introducing $h_{2}=h_{1}-d_{r} h_{0} / 2$, the evolution of the linear gas system is eventually described by $h_{0}$ and $h_{2}$, whose governing equations are written as 37 .

$$
\begin{aligned}
& \frac{\partial h_{0}}{\partial t}+\boldsymbol{v} \cdot \frac{\partial h_{0}}{\partial \boldsymbol{x}}=\mathcal{C}_{0}, \\
& \frac{\partial h_{2}}{\partial t}+\boldsymbol{v} \cdot \frac{\partial h_{2}}{\partial \boldsymbol{x}}=\mathcal{C}_{2},
\end{aligned}
$$

where the collision operators $\mathcal{C}_{0}$ and $\mathcal{C}_{2}$ take the forms

$$
\begin{array}{r}
\mathcal{C}_{0}=\mathcal{L}_{\mathrm{BCO}}+\frac{F_{e q}}{Z \tau}\left[\left(T-T_{t}\right)\left(|\boldsymbol{v}|^{2}-\frac{3}{2}\right)+\frac{4\left(\omega_{0}-1\right)}{15} \boldsymbol{q}_{t} \cdot \boldsymbol{v}\left(|\boldsymbol{v}|^{2}-\frac{5}{2}\right)\right], \\
\mathcal{C}_{2}=\frac{1}{\tau}\left(\frac{d_{r}}{2} T_{r} F_{e q}-h_{2}\right)+\frac{2\left(Z+\omega_{1}-1\right)(1-\delta)}{Z \tau} \boldsymbol{q}_{r} \cdot \boldsymbol{v} F_{e q}+\frac{d_{r}}{2 Z \tau}\left(T-T_{r}\right) F_{e q} .
\end{array}
$$

Here $\mathcal{L}_{\mathrm{BCO}}$ is the linearized Boltzmann collision operator [38], which models the elastic collision that conserves the kinetic energy. $\mathcal{L}_{\mathrm{BCO}}$ is a function of $F_{e q}, h_{0}$, and the intermolecular potential; we consider the inverse power-law potential, where the shear viscosity is a single power-law function of temperature, i.e. $\mu \propto T_{t}^{\omega}$ with $\omega$ the viscosity index and $T_{t}$ the translational temperature [39]. The other terms in $\mathcal{C}_{0}$ and $\mathcal{C}_{2}$ are the inelastic collision operators that describe the energy exchanges between translational and rotational motions, where $T, T_{r}, \boldsymbol{q}_{t}$ and $\boldsymbol{q}_{r}$ are the overall and rotational temperatures, heat fluxes related to the translational and rotational motions, respectively; $\tau$ is the mean collision time and $Z$ is the rotational collision number, such that the translational and rotational temperatures relax towards the overall temperature as $\partial T_{t} / \partial t=\left(T-T_{t}\right) / Z \tau$ and $\partial T_{r} / \partial t=\left(T-T_{r}\right) / Z \tau$, respectively. These energy exchanges are the origin of bulk viscosity that is absent in dilute monatomic gas. $\delta$ is the Schmidt number defined as the ratio of kinematic viscosity and mass diffusivity; it appears as the transport of internal energy occurs mainly due to the diffusion of gas molecules. The value of $\delta$ dependents on the intermolecular potential and usually in the range of $1 / 1.2$ to $1 / 1.55$ when the viscosity index $\omega$ varies from 0.5 to 1 . $\omega_{0}$ and $\omega_{1}$ relate to the translational $\left(\kappa_{t}\right)$ and rotational $\left(\kappa_{r}\right)$ heat conductivities which, according to the Chapman-Enskog expansion [40], are given as follows

$$
\begin{aligned}
& \boldsymbol{q}_{t}=-\frac{15}{8}\left(1+\frac{1-\omega_{0}}{2 Z}\right)^{-1} \tau \nabla T \equiv-\kappa_{t} \tau \nabla T \\
& \boldsymbol{q}_{r}=-\frac{d_{r}}{4}\left[\delta+\frac{\left(1-\omega_{1}\right)(1-\delta)}{Z}\right]^{-1} \tau \nabla T \equiv-\kappa_{r} \tau \nabla T .
\end{aligned}
$$


The macroscopic quantities of interest are flow density $\rho$, bulk velocity $\boldsymbol{U}$, stress tensor $\sigma_{i j}$, translational (rotational) temperature $T_{t}\left(T_{r}\right)$, and translational (rotational) heat flux $\boldsymbol{q}_{t}\left(\boldsymbol{q}_{r}\right)$, which are obtained from the velocity moments of the reduced velocity distribution functions as

$$
\begin{array}{r}
\rho=\int h_{0} \mathrm{~d} \boldsymbol{v}, \quad \boldsymbol{U}=\int \boldsymbol{v} h_{0} \mathrm{~d} \boldsymbol{v}, \quad \sigma_{i j}=2 \int\left(v_{i} v_{j}-\frac{|\boldsymbol{v}|^{2}}{3} \delta_{i j}\right) h_{0} \mathrm{~d} \boldsymbol{v} \\
T_{t}=\int\left(\frac{2}{3}|\boldsymbol{v}|^{2}-1\right) h_{0} \mathrm{~d} \boldsymbol{v}, \quad T_{r}=\frac{2}{d_{r}} \int h_{2} \mathrm{~d} \boldsymbol{v} \\
\boldsymbol{q}_{t}=\int \boldsymbol{v}\left(|\boldsymbol{v}|^{2}-\frac{5}{2}\right) h_{1} \mathrm{~d} \boldsymbol{v}, \quad \boldsymbol{q}_{r}=\int \boldsymbol{v} h_{2} \mathrm{~d} \boldsymbol{v}
\end{array}
$$

where $\delta_{i j}$ is the Kronecker delta function and $i, j=1,2,3$ represent the three orthogonal directions in the Cartesian coordinates. The overall temperature is the weighted sum of the translational and rotational temperatures: $T=\left(3 T_{t}+d_{r} T_{r}\right) /\left(3+d_{r}\right)$.

Dimensionless variables have been used in the above equations: $\boldsymbol{x}$ is normalized by the characteristic flow length $H ; \boldsymbol{v}$ and $\boldsymbol{U}$ are normalized by the most probable speed of gas molecules $v_{m}=\sqrt{2 k_{\mathrm{B}} T_{0} / m}$ with $k_{\mathrm{B}}$ being the Boltzmann constant, $T_{0}$ the reference temperature, and $m$ the molecular mass; $t$ is normalized by $H / v_{m} ; F_{e q}$ and $h_{0}$ are normalized by $n_{0} / v_{m}^{3}$, and $h_{2}$ is normalized by $n_{0} k_{B} T_{0} / v_{m}^{3}$, with $n_{0}$ being the reference number density of gas molecules; $\rho$ is normalized by $n_{0} ; \sigma_{i j}$ is normalized by $n_{0} k_{\mathrm{B}} T_{0}$; temperatures are normalized by $T_{0}$; heat fluxes are normalized by $n_{0} k_{\mathrm{B}} T_{0} v_{m}$. Finally the mean collision time is proportional to the Knudsen number as

$$
\tau=\frac{2 K n}{\sqrt{\pi}}=\frac{\mu\left(T_{0}\right) v_{m}}{n_{0} k_{\mathrm{B}} T_{0} H}
$$

with $\mu\left(T_{0}\right)$ the shear viscosity of gas at the reference temperature. In the following context, we will use $\tau$ to characterize the degree of rarefaction of gas flow.

In rarefied gas dynamics, turbulent flow is usually absent and steady-state solutions are of particular interest. The following iterative scheme is commonly adopted to find the steady-state solution of Eq. (3): given the values of $h_{0}^{k}$ and $h_{2}^{k}$ at the $k$-th iteration step, their values at the next iteration step are calculated by solving

$$
\frac{h_{s}^{k+1}}{\Delta t}+\boldsymbol{v} \cdot \frac{\partial h_{s}^{k+1}}{\partial \boldsymbol{x}}=\mathcal{C}_{s}^{k}+\frac{h_{s}^{k}}{\Delta t}, \quad s=0,2
$$

where $\Delta t$ is the iterative time step. This scheme can be understood as applying the backward Euler method for the time derivative with a constant step $\Delta t$, nevertheless the collision operators $\mathcal{C}_{s}^{k}$ are calculated at the $k$-th step rather than the $(k+1)$-th due to their complexity. When evaluating the collision operators, we use the fast spectral method to approximate the linearized Boltzmann collision operator $\mathcal{L}_{\mathrm{BCO}}$, and use the discrete velocity method to calculate the other parts [38, 39, 41].

We refer the iterative scheme (8) as CIS. It will be rigorously proven in Section 4 that the CIS is efficient for highly rarefied gas flow when the Knudsen number is large, however, it converges slowly when the Knudsen number approaches to zero. Worse still, the obtained solution suffers large numerical dissipation if the cell size of the spatial grid is much larger than the mean free path of gas molecules.

\section{General synthetic iterative scheme for molecular gas}

We have proposed the GSIS for monatomic gases to improve the CIS, which is particularly inefficient in simulating low Knudsen number flows. Here, we extend the GSIS to non-vibrational molecular gases, where energy exchange between the translational and rotational modes pose additional difficulties. In this section, we mainly describe the formulation and procedure of GSIS and left the analysis of its fast convergence and asymptotic preserving in the next sections. 
Given the values of $h_{0}^{k}$ and $h_{2}^{k}$ at the $k$-th step, the GSIS first calculates intermediate solutions of the reduced velocity distribution functions from the kinetic equations as the CIS does, read as

$$
\frac{h_{s}^{k+1 / 2}}{\Delta t}+\boldsymbol{v} \cdot \frac{\partial h_{s}^{k+1 / 2}}{\partial \boldsymbol{x}}=\mathcal{C}_{s}^{k}+\frac{h_{s}^{k}}{\Delta t}, \quad s=0,2 .
$$

Then from $h_{s}^{k+1 / 2}$, the GSIS finds the solutions at the $(k+1)$-th step by

$$
\begin{array}{r}
h_{0}^{k+1}=h_{0}^{k+1 / 2}+\left[\Delta \rho+2 \Delta \boldsymbol{U} \cdot \boldsymbol{v}+\Delta T_{t}\left(|\boldsymbol{v}|^{2}-\frac{3}{2}\right)\right] F_{e q}, \\
h_{2}^{k+1}=h_{2}^{k+1 / 2}+\left[\frac{d_{r}}{2} \Delta T_{r}+2 \Delta \boldsymbol{q}_{r} \cdot \boldsymbol{v}\right] F_{e q},
\end{array}
$$

where the quantities $\Delta M$ with $M=\left\{\rho, \boldsymbol{U}, T_{t}, T_{r}\right\}$ are defined as

$$
\Delta M=\bar{M}-M^{k+1 / 2} .
$$

Here $M^{k+1 / 2}$ are the ones calculated from $h_{0}^{k+1 / 2}$ and $h_{2}^{k+1 / 2}$ according to Eq. (6), and $\bar{M}$ can be considered as posterior correctors, such that $M^{k+1}=\bar{M}$ when evaluating them from $h_{0}^{k+1}$ and $h_{2}^{k+1}$. Since we aim to facilitate fast convergence and remove the restriction on spatial cell size in the CIS, $\bar{M}$ should be 'closer' to the final steady-state solution and more accurate on coarse mesh, compared to $M^{k+1 / 2}$. To this end, we propose to obtain $\bar{M}$ by solving the following synthetic equations (the Einstein summation is used)

$$
\begin{array}{r}
\frac{\partial \bar{\rho}}{\partial t}+\frac{\partial \bar{U}_{j}}{\partial x_{j}}=0, \\
2 \frac{\partial \bar{U}_{i}}{\partial t}+\frac{\partial \bar{\rho}}{\partial x_{i}}+\frac{\partial \bar{T}_{t}}{\partial x_{i}}+\frac{\partial \bar{\sigma}_{i j}}{\partial x_{j}}=0, \\
\frac{3}{2} \frac{\partial \bar{T}_{t}}{\partial t}+\frac{\partial \bar{U}_{j}}{\partial x_{j}}+\frac{\partial \bar{q}_{t, j}}{\partial x_{j}}=\frac{3}{2} \frac{\bar{T}-\bar{T}_{t}}{Z \tau}, \\
\frac{d_{r}}{2} \frac{\partial \bar{T}_{r}}{\partial t}+\frac{\partial \bar{q}_{r, j}}{\partial x_{j}}=\frac{d_{r}}{2} \frac{\bar{T}-\bar{T}_{r}}{Z \tau},
\end{array}
$$

where the time derivative is omitted in the real calculations since we are interested in steady-state solution. The macroscopic equations are exactly the conservation laws for mass, momentum and energy, which are strictly derived from the kinetic equations by firstly multiplying $1, v_{i}$ and $2|\boldsymbol{v}|^{2} / 3-1$ to Eq. (3a), respectively, and $2 / d_{r}$ to Eq. $3 \mathrm{~b}$ ), and then integrating the resultant equations in the molecular velocity space. The equations are not closed, since expressions for the stress and heat fluxed are still unknown. We have found that it is necessary to express the constitutive relations as 35

$$
\begin{gathered}
\bar{\sigma}_{i j}=-2 \tau \frac{\partial \bar{U}_{<i}}{\partial x_{j>}}+\operatorname{HoT}_{\sigma_{i j}}, \\
\bar{q}_{t, i}=-\kappa_{t} \tau \frac{\partial \bar{T}_{t}}{\partial x_{i}}+\operatorname{HoT}_{q_{t, i}}, \\
\bar{q}_{r, i}=-\kappa_{t} \tau \frac{\partial \bar{T}_{r}}{\partial x_{i}}+\operatorname{HoT}_{q_{r, i}},
\end{gathered}
$$

where $\frac{\partial \bar{U}_{<i}}{\partial x_{j}>}=\frac{1}{2}\left(\frac{\partial \bar{U}_{i}}{\partial x_{j}}+\frac{\partial \bar{U}_{j}}{\partial x_{i}}-\frac{2}{3} \frac{\partial \bar{U}_{k}}{\partial x_{k}} \delta_{i j}\right) ; \operatorname{HoT}_{\sigma_{i j}}, \operatorname{HoT}_{q_{t, i}}$ and $\operatorname{HoT}_{q_{r, i}}$ are the high-order terms rather than the first-order derivatives of the macroscopic quantities. It can be seen that Eqs. (12) and (13) reduce to the Navier-Stokes equations for molecular gas when the high-order terms vanish. 
To formulate the high-order terms is one of the most important parts when developing the GSIS. As we target dilute gas flows that might fall into any flow regime, the high-order terms should contain all the rarefied effects beyond the Navier-Stokes limit when Eq. (13) holds. We derive the high-order terms through the high-order moment equations of the kinetic equations. Taking Eq. (13a) for instance, we multiply Eq. (3a) by $2\left(v_{i} v_{j}-\delta_{i j}|\boldsymbol{v}|^{2} / 3\right)$ and integrate the resultant equation with respect to $\boldsymbol{v}$, then we obtain (the term of time derivative is eliminated)

$$
2 \int\left(v_{i} v_{j}-\frac{|\boldsymbol{v}|^{2}}{3} \delta_{i j}\right) \boldsymbol{v} \cdot \frac{\partial h_{0}}{\partial \boldsymbol{x}} \mathrm{d} \boldsymbol{v}=2 \int\left(v_{i} v_{j}-\frac{|\boldsymbol{v}|^{2}}{3} \delta_{i j}\right) \mathcal{C}_{0} \mathrm{~d} \boldsymbol{v}
$$

In order to obtain Eq. 13a, the term $\sigma_{i j} / \tau+2 \frac{\partial U_{<i}}{\partial x_{j}>}$ is added to the left and right hand sides of (14) respectively, written as

$$
2 \int\left(v_{i} v_{j}-\frac{|\boldsymbol{v}|^{2}}{3} \delta_{i j}\right) \boldsymbol{v} \cdot \frac{\partial h_{0}}{\partial \boldsymbol{x}} \mathrm{d} \boldsymbol{v}+\frac{\sigma_{i j}}{\tau}+2 \frac{\partial U_{<i}}{\partial x_{j>}}=\frac{\sigma_{i j}}{\tau}+2 \frac{\partial U_{<i}}{\partial x_{j>}}+2 \int\left(v_{i} v_{j}-\frac{|\boldsymbol{v}|^{2}}{3} \delta_{i j}\right) \mathcal{C}_{0} \mathrm{~d} \boldsymbol{v} .
$$

Thus $\mathrm{HoT}_{\sigma_{i j}}$ is devised as

$$
\mathrm{HoT}_{\sigma_{i j}}=2 \tau\left[\frac{\partial U_{<i}}{\partial x_{j>}}-\int\left(v_{i} v_{j}-\frac{|\boldsymbol{v}|^{2}}{3} \delta_{i j}\right) \boldsymbol{v} \cdot \frac{\partial h_{0}}{\partial \boldsymbol{x}} \mathrm{d} \boldsymbol{v}\right]+\sigma_{i j}+2 \tau \int\left(v_{i} v_{j}-\frac{|\boldsymbol{v}|^{2}}{3} \delta_{i j}\right) \mathcal{C}_{0} \mathrm{~d} \boldsymbol{v}
$$

Similarly, we multiply Eq. (3a) with $v_{i}\left(|\boldsymbol{v}|^{2}-5 / 2\right)$ and Eq. $3 \mathrm{~b}$ with $v_{i}$, and integrate the resultant equations with respect to $\boldsymbol{v}$, then we have

$$
\begin{array}{r}
\int v_{i}\left(|\boldsymbol{v}|^{2}-\frac{5}{2}\right) \boldsymbol{v} \cdot \frac{\partial h_{0}}{\partial \boldsymbol{x}} \mathrm{d} \boldsymbol{v}=\int v_{i}\left(|\boldsymbol{v}|^{2}-\frac{5}{2}\right) \mathcal{C}_{0} \mathrm{~d} \boldsymbol{v} \\
\int v_{i} \boldsymbol{v} \cdot \frac{\partial h_{2}}{\partial \boldsymbol{x}} \mathrm{d} \boldsymbol{v}=\int v_{i} \mathcal{C}_{2} \mathrm{~d} \boldsymbol{v}
\end{array}
$$

which are rearranged as

$$
\begin{array}{r}
\int v_{i}\left(|\boldsymbol{v}|^{2}-\frac{5}{2}\right) \boldsymbol{v} \cdot \frac{\partial h_{0}}{\partial \boldsymbol{x}} \mathrm{d} \boldsymbol{v}+\frac{5}{4 \kappa_{t} \tau} q_{t, i}+\frac{5}{4} \frac{\partial T_{t}}{\partial x_{i}}=+\frac{5}{4 \kappa_{t} \tau} q_{t, i}+\frac{5}{4} \frac{\partial T_{t}}{\partial x_{i}}+\int v_{i}\left(|\boldsymbol{v}|^{2}-\frac{5}{2}\right) \mathcal{C}_{0} \mathrm{~d} \boldsymbol{v} \\
\int v_{i} \boldsymbol{v} \cdot \frac{\partial h_{2}}{\partial \boldsymbol{x}} \mathrm{d} \boldsymbol{v}+\frac{d_{r}}{4 \kappa_{r} \tau} q_{r, i}+\frac{d_{r}}{4} \frac{\partial T_{r}}{\partial x_{i}}=\frac{d_{r}}{4 \kappa_{r} \tau} q_{r, i}+\frac{d_{r}}{4} \frac{\partial T_{r}}{\partial x_{i}}+\int v_{i} \mathcal{C}_{2} \mathrm{~d} \boldsymbol{v}
\end{array}
$$

Therefore, the high-order terms $\operatorname{HoT}_{q_{t, i}}$ and $\operatorname{HoT}_{q_{r, i}}$ have the following forms:

$$
\begin{array}{r}
\operatorname{HoT}_{q_{t, i}}=\kappa_{t} \tau\left[\frac{\partial T_{t}}{\partial x_{i}}-\frac{4}{5} \int v_{i}\left(|\boldsymbol{v}|^{2}-\frac{5}{2}\right) \boldsymbol{v} \cdot \frac{\partial h_{0}}{\partial \boldsymbol{x}} \mathrm{d} \boldsymbol{v}\right]+q_{t, i}+\frac{4 \kappa_{t} \tau}{5} \int v_{i}\left(|\boldsymbol{v}|^{2}-\frac{5}{2}\right) \mathcal{C}_{0} \mathrm{~d} \boldsymbol{v} \\
\operatorname{HoT}_{q_{r, i}}=\kappa_{r} \tau\left[\frac{\partial T_{r}}{\partial x_{i}}-\frac{4}{d_{r}} \int v_{i} \boldsymbol{v} \cdot \frac{\partial h_{2}}{\partial \boldsymbol{x}} \mathrm{d} \boldsymbol{v}\right]+q_{r, i}+\frac{4 \kappa_{r} \tau}{d_{r}} \int v_{i} \mathcal{C}_{2} \mathrm{~d} \boldsymbol{v}
\end{array}
$$

According to the property of the linearized Boltzmann collision operator, the last two terms in each highorder expressions are very close to zero 33,40 . Especially, when $\mathcal{L}_{\mathrm{BCO}}$ is replaced by the one from the Shakhov kinetic model [42, the kinetic equations become the Rykov kinetic model [20], where the last two terms vanish.

We summarize the procedures of GSIS for molecular gases to close this section:

- Step 1. When the velocity distribution functions $h_{s}^{k}$ and the corresponding macroscopic quantities in Eq. (6) are known at the $k$-th iteration, we calculate the Boltzmann collision operator $\mathcal{L}_{\mathrm{BCO}}$ in Eq. (4). We also calculate the intermediate solutions $h_{s}^{k+1 / 2}$ according to Eq. (9). 
- Step 2. From $h_{s}^{k+1 / 2}$, we calculate the macroscopic quantities $M^{k+1 / 2}$ and then the high-order terms $\mathrm{HoT}_{\sigma_{i j}}, \mathrm{HoT}_{q_{t, i}}$ and $\mathrm{HoT}_{q_{r, i}}$ defined in Eqs. (16) and (19). Note that when calculating $\mathrm{HoT}_{\sigma_{i j}}$, we use the $\mathcal{L}_{\mathrm{BCO}}$ obtained in Step 1 from $h_{s}^{k}$, since its computational cost by the fast spectral method is relatively high and we only evaluate it once during each iteration.

- Step 3. We obtain the macroscopic quantities $\bar{M}$ by solving the synthetic equations for the conservation laws (12), where the shear stress and heat fluxes are determined by the Navier-Stokes-like constitutive relations (13) with source terms, i.e. the high-order terms that take account all the rarefaction effects.

- Step 4 . The solutions $h_{s}^{k+1}$ at the $(k+1)$-th iterative step are obtained by incorporate the change of macroscopic quantities, according to Eq. 10 .

- Step 5. The above steps are repeated until convergence.

\section{Fast convergence and asymptotic preserving of GSIS}

This section is dedicated to analyzing the convergence rate of CIS and GSIS rigorously, as well as the asymptotic preserving property of GSIS for the simulation of molecular gas flows. To make the calculation tractable, the linearized Boltzmann collision operator $\mathcal{L}_{\mathrm{BCO}}$ in Eq. $3 \mathrm{a}$ is replaced by the Shakhov kinetic model for monatomic gas 42

$$
\mathcal{L}_{\mathrm{S}}=\frac{F_{e q}}{\tau}\left[\rho+2 \boldsymbol{U} \cdot \boldsymbol{v}+T_{t}\left(|\boldsymbol{v}|^{2}-\frac{3}{2}\right)+\frac{4}{15} \boldsymbol{q}_{t} \cdot \boldsymbol{v}\left(|\boldsymbol{v}|^{2}-\frac{5}{2}\right)\right]-\frac{h_{0}}{\tau},
$$

then the kinetic system is exactly reduced to the Rykov model [20]. The streaming operator $\partial_{\boldsymbol{x}}$ is kept intact when calculating the convergence rate; while the convergence rate of iteration for the system discretized over a computational grid will be shown numerically in Section 5 Also, for simplicity we assume the system varies only in the $x_{1}-x_{2}$ plane, which keeps the essential physics but simplifies the computation.

\subsection{Convergence rate of CIS}

First, we define the error functions of the velocity distribution functions between two consecutive iteration steps

$$
Y_{s}^{k+1}(\boldsymbol{x}, \boldsymbol{v})=h_{s}^{k+1}(\boldsymbol{x}, \boldsymbol{v})-h_{s}^{k}(\boldsymbol{x}, \boldsymbol{v}), \quad s=0,2,
$$

and the error functions for macroscopic quantities $M_{0}=\left[\rho, U_{1}, U_{2}, T_{t}, q_{t, 1}, q_{t, 2}\right]$ and $M_{2}=\left[T_{r}, q_{r, 1}, q_{r, 2}\right]$ between two consecutive iteration steps

$$
\Phi_{M_{s}}^{k+1}(\boldsymbol{x})=M_{s}^{(k+1)}(\boldsymbol{x})-M_{s}^{(k)}(\boldsymbol{x})=\int Y_{s}^{k+1}(\boldsymbol{x}, \boldsymbol{v}) \phi_{s}(\boldsymbol{v}) d \boldsymbol{v},
$$

where

$$
\phi_{0}(\boldsymbol{v})=\left[1, v_{1}, v_{2}, \frac{2}{3}|\boldsymbol{v}|^{2}-1, v_{1}\left(|\boldsymbol{v}|^{2}-\frac{5}{2}\right), v_{2}\left(|\boldsymbol{v}|^{2}-\frac{5}{2}\right)\right], \quad \phi_{2}(\boldsymbol{v})=\left[\frac{2}{d_{r}}, v_{1}, v_{2}\right] .
$$

Second, to determine the convergence rate $g$ we perform the Fourier stability analysis by seeking the eigenfunctions $y_{s}(\boldsymbol{v}), \alpha_{M_{0}}=\left[\alpha_{\rho}, \alpha_{U_{1}}, \alpha_{U_{2}}, \alpha_{T_{t}}, \alpha_{q_{t, 1}}, \alpha_{q_{t, 2}}\right]$ and $\alpha_{M_{2}}=\left[\alpha_{T_{r}}, \alpha_{q_{r, 1}}, \alpha_{q_{r, 2}}\right]$ of the following forms

$$
Y_{s}^{k+1}(\boldsymbol{x}, \boldsymbol{v})=g^{k} y_{s}(\boldsymbol{v}) \exp (\imath \boldsymbol{\theta} \cdot \boldsymbol{x}), \quad \Phi_{M_{s}}^{k+1}(\boldsymbol{x})=g^{k+1} \alpha_{M_{s}} \exp (\imath \boldsymbol{\theta} \cdot \boldsymbol{x}),
$$

where $\imath$ is the imaginary unit and $\boldsymbol{\theta}=\left(\theta_{1}, \theta_{2}\right)$ is the wave vector of perturbation. The slow convergence occurs when $|g|$ approaches one, where the errors barely reduce during iteration, while the fast convergence is realized when $|g|<1$, especially when $|g|$ approaches zero. 
Here we consider the case that the iterative step $\Delta t=\tau$ and denote the corresponding convergence rate as $g_{0}$. The convergence rate for other values of $\Delta t$ (not larger than $\tau$ to make the iteration stable) is obtained as

$$
|g|=g_{0}^{\Delta t / \tau}
$$

considering the fact that the velocity distribution functions in Eq. (3) decay with a fixed rate irrespective of $\Delta t$ appearing in Eq. (8).

Obviously, from Eqs. 222) and (24) we have

$$
g_{0} \alpha_{M_{s}}=\int y_{s}(\boldsymbol{v}) \phi_{s}(\boldsymbol{v}) \mathrm{d} \boldsymbol{v},
$$

and from Eqs. (4), (8), 20, 21), and (24), we obtain the following expressions for $y_{s}(\boldsymbol{v})$

$$
\begin{gathered}
(1+\imath \tau \boldsymbol{\theta} \cdot \boldsymbol{v}) y_{0}=\left(\alpha_{\rho}+2 \alpha_{U_{1}} v_{1}+2 \alpha_{U_{2}} v_{2}\right) F_{e q}+\left[\frac{d_{r}\left(\alpha_{T_{r}}-\alpha_{T_{t}}\right)}{Z\left(d_{r}+3\right)}+\alpha_{T_{t}}\right]\left(|\boldsymbol{v}|^{2}-\frac{3}{2}\right) F_{e q} \\
+\frac{4}{15}\left[1+\frac{\left(\omega_{0}-1\right)}{Z}\right]\left(|\boldsymbol{v}|^{2}-\frac{5}{2}\right)\left(\alpha_{q_{\mathrm{t}, 1}} v_{1}+\alpha_{q_{\mathrm{t}, 2}} v_{2}\right) F_{e q}, \\
(1+\imath \tau \boldsymbol{\theta} \cdot \boldsymbol{v}) y_{2}= \\
\frac{d_{r}}{2} \alpha_{T_{r}} F_{e q}+\frac{3 d_{r}}{2 Z\left(d_{r}+3\right)}\left(\alpha_{T_{t}}-\alpha_{T_{r}}\right) F_{e q} \\
+\frac{2\left(Z+\omega_{1}-1\right)(1-\delta)}{Z}\left(\alpha_{q_{r, 1}} v_{1}+\alpha_{q_{r, 2}} v_{2}\right) F_{e q} .
\end{gathered}
$$

Note that here we assume the wave vector of perturbation satisfies $|\boldsymbol{\theta}|^{2}=\theta_{1}^{2}+\theta_{2}^{2}=1$. Although in reality the perturbation may have various values of $\boldsymbol{\theta}$, their corresponding convergence rates do not interact because the kinetic equations are linear. Moreover it can be concluded that the convergence rate depends only on the product of $\tau \boldsymbol{\theta}$, thus it is safe to assume $|\boldsymbol{\theta}|=1$. If $|\boldsymbol{\theta}| \neq 1$, the convergence rate at specific values of $\boldsymbol{\theta}$ and $\tau$ can be calculated by replacing $\tau$ with $\tau|\boldsymbol{\theta}|$.

Finally, multiplying Eq. (27a) with $\phi_{0}(\boldsymbol{v})$ and Eq. $\left.27 \mathrm{~b}\right)$ with $\phi_{2}(\boldsymbol{v})$, and integrating the resultant equation with respect to $\boldsymbol{v}$, we obtain nine linear algebraic equations for unknowns $\alpha_{M_{s}}$ with the aid of Eqs. (26). These algebraic equations can be written in the matrix form as

$$
\mathbf{C} \alpha_{M}^{\top}=g_{0} \alpha_{M}^{\top}, \quad \text { with } \alpha_{M}=\left[\alpha_{M_{0}}, \alpha_{M_{2}}\right],
$$

where the superscript $T$ is the transpose operator. The details of the coefficient matrix $\mathbf{C}$ of $9 \times 9$ dimension are given in the Appendix A. In general, the convergence rate can be obtained by numerically computing the eigenvalues $g_{0}$ of matrix $\mathbf{C}$ and taking the maximum absolute value of $g_{0}$. The result of convergence rate as a function of the Knudsen number $(\tau)$ for CIS is shown as the red solid line in Figure 2. It is clear that when the Knudsen number is large, $g_{0}$ goes to zero so that the error between iterations decays quickly. On the other hand, $g_{0} \rightarrow 1$ when $\tau \rightarrow 0$, which means it is hard to obtain converged steady-state solutions in the near-continuum flow regime.

\subsection{Convergence rate of GSIS}

To calculate the convergence rate of GSIS for molecular gas, the error functions in Eqs. [21, 22, , and 240) are redefined as

$$
\begin{gathered}
Y_{s}^{k+1 / 2}(\boldsymbol{x}, \boldsymbol{v})=h_{s}^{k+1 / 2}(\boldsymbol{x}, \boldsymbol{v})-h_{s}^{k}(\boldsymbol{x}, \boldsymbol{v})=g_{0}^{k} y_{s}(\boldsymbol{v}) \exp (\imath \boldsymbol{\theta} \cdot \boldsymbol{x}), \\
\Phi_{M}^{k+1}(\boldsymbol{x})=M^{k+1}(\boldsymbol{x})-M^{k}(\boldsymbol{x})=g_{0}^{k+1} \alpha_{M} \exp (\imath \boldsymbol{\theta} \cdot \boldsymbol{x}),
\end{gathered}
$$

where the solutions of $y_{s}(\boldsymbol{v})$ are still given by Eq. 27). Note that the definitions for $\Phi_{M_{s}}$ remain unchanged, but in the GSIS they will be calculated from the solutions of macroscopic synthetic equations, rather than 


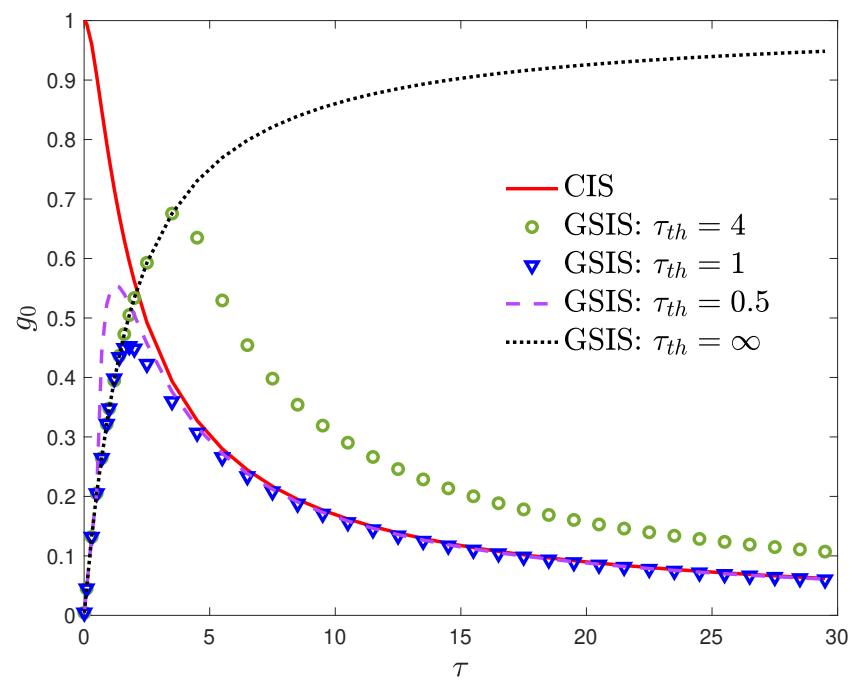

Figure 2: The convergence rate $g_{0}$ as a function of the Knudsen number $\tau$ in both CIS and GSIS. $\tau_{t h}$ is the threshold Knudsen number used to define the relaxation parameter in Eq. (31). Here $g_{0}$ are obtained under $d_{r}=2.0, Z=1.0, \delta=1 / 1.33, \omega_{0}=1.5$ and $\omega_{1}=0.5$.

directly from $Y_{s}$. On substituting Eqs. 29) into Eqs. (12) and (13), we obtain a system of linear algebraic equations for $\alpha_{M}$ that can be written in the following matrix form

$$
\mathbf{L} g_{0} \alpha_{M}^{\top}=\mathbf{R} \alpha_{M}^{\top},
$$

where both $\mathbf{L}$ and $\mathbf{R}$ are $9 \times 9$ matrices, the detailed expressions of which are as well presented in Appendix A. By introducing $\mathbf{G}=\mathbf{L}^{-1} \mathbf{R}$ and numerically computing the eigenvalues of $\mathbf{G}$ we obtain the convergence rate $g_{0}$ of GSIS, see the black dashed line in Figure 2 It is shown that $g_{0}$ goes to zero when $\tau \rightarrow 0$, which demonstrates that the GSIS is able to boost convergence rate significantly in the near-continuum flow regime. However, the convergence rate increases to one when $\tau \rightarrow \infty$, i.e. the convergence of GSIS becomes slow as the Knudsen number becomes large. To fix this problem and achieve fast convergence at any Knudsen number, we introduce a relaxation parameter $\beta$ when correcting the velocity distribution functions at $(k+1)$-th step in Eq. (10) and now Eq. (11) becomes

$$
\Delta M=\beta\left[\bar{M}-M^{k+1 / 2}\right], \quad \beta=\frac{\min \left(\tau, \tau_{\mathrm{th}}\right)}{\tau},
$$

where $\tau_{\text {th }}$ is a threshold Knudsen number. The convergence rate of this GSIS can be obtained by computing the eigenvalue of the matrix $\mathbf{G}=\beta \mathbf{L}^{-1} \mathbf{R}+(1-\beta) \mathbf{C}$, where the result of $\tau_{t h}=\infty$ is reduced to the one for Eq. (30). The convergence rates at the threshold Knudsen number of values 0.5, 1 and 4 are shown in Figure 2. Clearly, $\beta$ cannot be too small nor too large. By choosing a proper value of $\beta$, we can make the maximum value of $g_{0}$ always less than 0.5 ; this means that after 10 iterations, the error will be decreased by at least three orders of magnitude. Thus, theoretically, the GSIS can reach fast convergence in the entire flow regime.

\subsection{Asymptotic Navier-Stokes preserving of GSIS}

In the CIS the spatial cell size has to be very small (nearly at the order of mean free path) in order to find accurate numerical solution that is not contaminated by the numerical dissipation (e.g. numerical viscosity). This makes the number of spatial cells extremely huge for the CIS when simulating near-continuum flow. Now we analyze whether the GSIS can circumvent this problem. To be specific, we investigate whether the 
proposed synthetic equations is able to recover the Navier-Stokes equations for molecular gas in the limit of $\tau \rightarrow 0$ (i.e. the high-order terms in Eq. (13) obtained from the distribution functions vanish) or not. For simplicity, let us take the Rykov model for example, i.e. the linearized Boltzmann collision operator $\mathcal{L}_{\mathrm{BCO}}$ in Eq. (3a) is replaced by Eq. 200.

To this end, we apply the Chapman-Enskog expansion, where the velocity distribution functions are approximated by the Taylor expansion [40.

$$
h_{s}=h_{s, 0}+\tau h_{s, 1}+\tau^{2} h_{s, 2}+\cdots .
$$

The expansion coefficients are determined by the following discretized version of kinetic equations:

$$
\boldsymbol{v} \cdot \frac{\partial h_{s}}{\partial \boldsymbol{x}}+O\left(\Delta x^{n}\right) \boldsymbol{v} \cdot \frac{\partial^{n+1} h_{s}}{\partial \boldsymbol{x}^{n+1}}=\mathcal{C}_{s}, \quad s=0,2,
$$

where the spatial derivative $\partial_{\boldsymbol{x}}$ is discretized by a $n$-th order scheme and $O\left(\Delta x^{n}\right) \partial_{\boldsymbol{x}}^{n+1} h_{s}$ is the leading term of the truncation error. Note that the solution does not depend on the time when the steady state is reached. Substituting Eq. (32) into Eq. (33), it is found that if choosing

$$
\Delta x=O\left(\tau^{0}\right)
$$

such that the spatial cell size is independent of the Knudsen number, we have (note that when $\tau$ approaches zero, $\beta=1$ as defined in Eq. (31)):

$$
\begin{aligned}
h_{0,0}= & F_{e q}\left[\rho+2 \boldsymbol{U} \cdot \boldsymbol{v}+T_{t}\left(|\boldsymbol{v}|^{2}-\frac{3}{2}\right)+\frac{4}{15} \boldsymbol{q}_{t} \cdot \boldsymbol{v}\left(|\boldsymbol{v}|^{2}-\frac{5}{2}\right)\right] \\
& +\frac{F_{e q}}{Z}\left[\left(T-T_{r}\right)\left(|\boldsymbol{v}|^{2}-\frac{3}{2}\right)+\frac{4\left(\omega_{0}-1\right)}{15} \boldsymbol{q}_{t} \cdot \boldsymbol{v}\left(|\boldsymbol{v}|^{2}-\frac{5}{2}\right)\right]
\end{aligned}
$$

and

$$
h_{2,0}=\frac{d_{r}}{2} T_{r} F_{e q}+\frac{2\left(Z+\omega_{1}-1\right)(1-\delta)}{Z} \boldsymbol{q}_{r} \cdot \boldsymbol{v} F_{e q}+\frac{d_{r}}{2 Z}\left(T-T_{r}\right) F_{e q} .
$$

Then, from the definition of high-order terms in Eqs. 16 and $\sqrt{19}$, we find that the constitutive relations can be recovered as

$$
\begin{array}{r}
\sigma_{i j}=-2 \tau \frac{\partial U_{<i}}{\partial x_{j}}+O\left(\tau^{2}\right), \\
q_{t, i}=-\kappa_{t} \tau \frac{\partial T_{t}}{\partial x_{i}}+O\left(\tau^{2}\right), \quad q_{r, i}=-\kappa_{r} \tau \frac{\partial T_{r}}{\partial x_{i}}+O\left(\tau^{2}\right) .
\end{array}
$$

Furthermore, from the Chapman-Enskog expansion of the last two equations in Eq. (12, we have $T_{r}=$ $T+O(\tau)$ and

$$
T_{t}=T-\frac{2 d_{r} Z}{3\left(d_{r}+3\right)} \tau+O\left(\tau^{2}\right)
$$

where the term $2 d_{r} Z / 3\left(d_{r}+3\right)$ is the ratio of the bulk viscosity to the shear viscosity. Then Eq. (38) becomes

$$
q_{t, i}=-\kappa_{t} \tau \frac{\partial T}{\partial x_{i}}+O\left(\tau^{2}\right), \quad q_{r, i}=-\kappa_{r} \tau \frac{\partial T}{\partial x_{i}}+O\left(\tau^{2}\right)
$$

and hence the Navier-Stokes equation for molecular gas can be recovered. This means that we can choose the spatial cell size that is much larger than the Knudsen number (mean free path) to recover the NavierStokes equations when $\tau \rightarrow 0$. Note that, since the macroscopic synthetic equations are also solved by a discrete scheme, in order to ensure the truncation error of the discretization is small, the spatial resolution $\Delta x=O\left(\tau^{0}\right)$ is required to be adequate to resolve the macroscopic flows. Note that the same conclusion can be obtained for the $\mathrm{Wu}$ et al. model [37]. 


\section{Numerical results and discussions}

To assess the performance of the proposed GSIS for rarefied molecular gas, we consider five different test cases, including one-dimensional thermal creep flow and conductive heat transfer between two parallel plates, two-dimensional thermal creep flow and conductive heat transfer inside a square cavity, and a thermal driven flow induced by a hot beam in a rectangular chamber. The linearized $\mathrm{Wu}$ et al. (3) as well as the Rykov model equations [where $\mathcal{L}_{\mathrm{BCO}}$ in Eq. (3a) is replaced by the Shakhov model (20p] are solved. The 4thorder discontinuous Galerkin (DG) method is employed to discretize the spatial derivatives (the details are given in Appendix B together with the implementation of different boundary conditions). The convergence criterion for the iterative schemes is that the maximum relative difference between successive estimates of the macroscopic quantities $M$, i.e.

$$
\epsilon=\max \left\{\mathcal{R}_{M}\right\}, \quad \mathcal{R}_{M}=\sqrt{\frac{\int\left|M^{k+1}-M^{k}\right|^{2} \mathrm{~d} \boldsymbol{x}}{\int\left|M^{k+1}\right|^{2} \mathrm{~d} \boldsymbol{x}}},
$$

is less than $10^{-5}$. The tests are performed in double precision on a workstation with Intel Xeon-E5-2680 processors and 128 GB RAM. We call the corresponding routines in Intel Math Kernel Library (MKL) to solve the linear systems arisen in the DG discretization and calculate FFT when evaluating $\mathcal{L}_{\mathrm{BCO}}$ if necessary.

\subsection{Thermal creep flows between two parallel plates}

Consider a molecular gas flow that is confined between two stationary parallel plates located at $x_{2}=0$ and $x_{2}=1$ and driven by a constant temperature gradient $K_{T}=1$ along the $x_{1}$ direction at $\tau \in[0.01,10]$, where the gas moves toward the hotter region in spite of uniform gas pressure everywhere. We assume that the solid plates extend to infinite in both the $x_{1}$ and $x_{3}$ directions, then the flow is essentially a one-dimensional problem and macroscopic flow quantities only vary with $x_{2}$. Due to the presence of a temperature gradient, additional terms appear in the governing system, which read as (only the modified equations are shown)

$$
\begin{array}{r}
\boldsymbol{v} \cdot \frac{\partial h_{0}}{\partial \boldsymbol{x}}=\mathcal{C}_{0}-v_{1}\left(|\boldsymbol{v}|^{2}-\frac{5}{2}\right) K_{T} F_{e q}, \\
\boldsymbol{v} \cdot \frac{\partial h_{2}}{\partial \boldsymbol{x}}=\mathcal{C}_{2}-v_{1} \frac{d_{r}}{2} K_{T} F_{e q}, \\
\bar{q}_{t, 1}+\kappa_{t} \tau \frac{\partial \bar{T}_{t}}{\partial x_{1}}=\mathrm{HoT}_{q_{t, 1}}-\kappa_{t} \tau K_{T}, \\
\bar{q}_{r, 1}+\kappa_{r} \tau \frac{\partial \bar{T}_{r}}{\partial x_{1}}=\mathrm{HoT}_{q_{r, 1}}-\kappa_{r} \tau K_{T} .
\end{array}
$$

The flow is simulated by the CIS and the GSIS on two-dimensional triangular mesh. The triangular cells are refined in the vicinity of walls (i.e. $0<x_{2}<0.08$ and $0.92<x_{2}<1$ ) to resolve the structure of Knudsen layers, in which the flow velocity varies significantly when the Knudsen number is small, see the schematic in Figure 3(a). The upper and lower boundaries are fully-diffuse wall, while the left and right boundaries are periodic. The Rykov kinetic model is solved by discrete velocity method, using $32 \times 32 \times 24$ discrete velocities over a truncated domain of $[-4,4]^{3}$, where $v_{1}$ and $v_{2}$ are discretized by non-uniform nodes

$$
v_{i}=\frac{v_{\max }}{\left(N_{v_{i}}-1\right)^{3}}\left(-N_{v_{i}}+1,-N_{v_{i}}+3, \cdots, N_{v_{i}}-1\right)^{3}, \quad i=1 \text { or } 2,
$$

with $N_{v_{i}}=32$ the number of discrete velocities and $v_{\max }=4$ the maximum molecular velocity, while $v_{3}$ is discretized by uniform nodes. The time step for iteration is $\Delta t=\tau$. We use the same gas parameters as those in Ref. [37: the viscosity index is $\omega=0.5$, the rotational degree of freedom is $d_{r}=2$, the rotational 
(a)

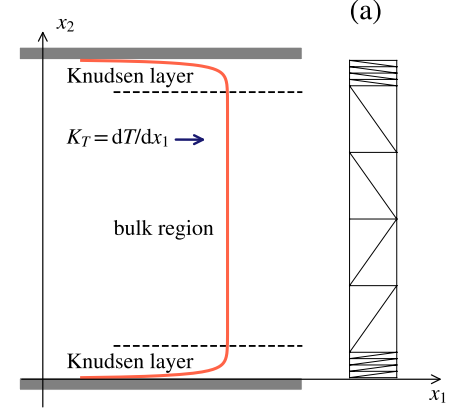

(b)

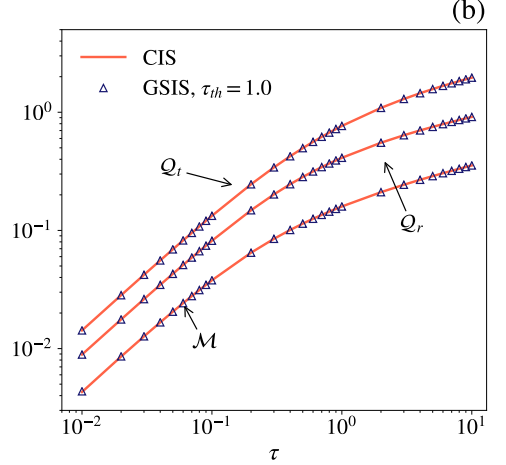

(c)

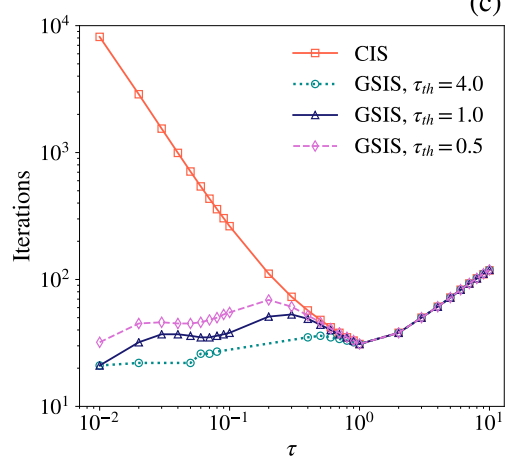

Figure 3: (a) Schematic of the thermal creep flow between two parallel plates, driven by a constant temperature gradient in the $x_{1}$ direction. The one-dimensional problem is solved on triangular mesh with refinement near the solid wall. Comparisons between GSIS and CIS over a range of Knudsen numbers $\tau \in[0.01,10]$ : (b) the mass flow rate $\mathcal{M}$ and translational (rotational) heat flow rate $\mathcal{Q}_{t}\left(\mathcal{Q}_{r}\right) ;(\mathrm{c})$ the number of iterations to reach the convergence criterion $\epsilon=\max \left\{\mathcal{R}_{U_{1}}, \mathcal{R}_{q_{t, 1}}, \mathcal{R}_{q_{r, 1}}\right\}<10^{-5}$ using $\Delta t=\tau$. The Rykov model is solved with gas properties of $d_{r}=2, \delta=1 / 1.33, Z=1.0, \omega_{0}=0.389$, and $\omega_{1}=1.787$.

collision number is $Z=1.0$, the Schmidt number is $\delta=1 / 1.33$, and $\omega_{0}=0.389$ and $\omega_{1}=1.787$ that are determined from the kinetic theory of Mason \& Monchick [43] by

$$
\begin{aligned}
& 1-\frac{5 d_{r}}{4 Z\left(3+d_{r}\right)}\left(1-\frac{2}{5 \delta}\right)=\left(1+\frac{1-\omega_{0}}{2 Z}\right)^{-1} \\
& 1+\frac{15}{4 Z\left(3+d_{r}\right)}\left(1-\frac{2}{5 \delta}\right)=\left(1+\frac{(1-\delta)\left(1-\omega_{1}\right)}{Z \delta}\right)^{-1}
\end{aligned}
$$

The 4th order DG method is applied to discretize the governing equations in the spatial space on 64 triangles (with 16 uniform triangles in each Knudsen layer and 32 uniform ones in the bulk region). The relaxation parameter $\beta$ in Eq. (31) for the correction of the velocity distributions in the GSIS is calculated as

$$
\beta=\frac{\min \left(\tau_{l o c}, \tau_{t h}\right)}{\tau_{l o c}},
$$

where $\tau_{l o c}$ is the local Knudsen number estimated from the local cell size, i.e. $\tau_{l o c}=\tau / H_{l o c}$ with $H_{l o c}$ the height of triangles, to include the local rarefaction effect (e.g. the local Knudsen number within the Knudsen layer is always larger than 1 ).

Figure $3(\mathrm{~b})$ shows the mass flow rate $\mathcal{M}=\int_{0}^{1} U_{1} \mathrm{~d} x_{2}$, the translational heat flow rate $\mathcal{Q}_{t}=\int_{0}^{1} q_{t, 1} \mathrm{~d} x_{2}$ and the rotational heat flow rate $\mathcal{Q}_{r}=\int_{0}^{1} q_{r, 1} \mathrm{~d} x_{2}$. Over the considered range of Knudsen numbers, the mass flow rate and the heat flux rates increase against the Knudsen number and the GSIS results are coincident with the CIS ones. Figure 3(c) shows the total number of iterations for the CIS and the GSIS to reach the convergence criterion $\epsilon=\max \left\{\mathcal{R}_{U_{1}}, \mathcal{R}_{q_{t, 1}}, \mathcal{R}_{q_{r, 1}}\right\}<10^{-5}$. When the Knudsen number $\tau$ drops from 1 to 0.01 , the number of iterations by the CIS significantly increase from about 30 to more than 8000; on the contrary, that of the GSIS is always below 70, and the GSIS only needs as few as 21 steps to reach the steady state solution at $\tau=0.01$. It is also found that, for the three threshold Knudsen numbers $\tau_{t h}$, larger $\tau_{t h}$ results in faster convergence. However, the iteration is not stable at some Knudsen numbers when $\tau_{t h}=4$. This is due to the fact that the high-order terms defined in Eqs. (16) and (19) may have strong variations within the Knudsen layer, which leads to unphysical solutions and blowups of the code when solving Eq. (12). In practice, we usually choose $\tau_{t h} \leq 1$ to keep the calculation stable. When $\tau>1$, the GSIS and the CIS use almost the same number of iterations, which increase slightly as the Knudsen number increases, since the relaxation parameter $\beta$ approaches to 0 when the Knudsen number goes to infinity and the behavior of the GSIS reduces to that of the CIS. 
Table 1: Comparison between GSIS and CIS for one-dimensional thermal creep flow in terms of the mass flow rate $\mathcal{M}$, the number of iterations (Itr) to reach $\epsilon=\max \left\{\mathcal{R}_{U_{1}}, \mathcal{R}_{q_{t, 1}}, \mathcal{R}_{q_{r, 1}}\right\}<10^{-5}$ with $\Delta t=\tau$ and $\tau_{t h}=1$ in GSIS, as well as the CPU time cost on single processor. The Rykov model is solved with $d_{r}=2, \delta=1 / 1.33, Z=1.0, \omega_{0}=0.389$, and $\omega_{1}=1.787$. The molecular velocity domain $[-4,4]^{3}$ is discretized by $32 \times 32 \times 24$ points and the spatial domain is discretized by 64 triangles. The 4th-order DG scheme is employed. The results obtained by a finite difference solver using the CIS [37] is listed in the last column for reference.

\begin{tabular}{|c|c|c|c|c|c|c|c|}
\hline \multirow[b]{2}{*}{$\tau$} & \multicolumn{3}{|c|}{ GSIS } & \multicolumn{3}{|c|}{ CIS } & \multirow{2}{*}{$\mathcal{M} 37$} \\
\hline & $\mathcal{M}$ & Itr & CPU time $[\mathrm{s}]$ & $\overline{\mathcal{M}}$ & Itr & CPU time $[\mathrm{s}]$ & \\
\hline 0.01 & 0.0044 & 21 & 80.9 & 0.0043 & 8158 & 27086.2 & - \\
\hline 0.125 & 0.0454 & 41 & 152.5 & 0.0454 & 196 & 645.5 & 0.0455 \\
\hline 1.0 & 0.1594 & 31 & 117.2 & 0.1594 & 31 & 105.5 & 0.1594 \\
\hline 10.0 & 0.3546 & 118 & 448.4 & 0.3546 & 118 & 371.2 & 0.3546 \\
\hline
\end{tabular}

In Table 1. we compare the GSIS and the CIS in terms of the mass flow rate, the number of iterations to reach $\epsilon=\max \left\{\mathcal{R}_{U_{1}}, \mathcal{R}_{q_{t, 1}}, \mathcal{R}_{q_{r, 1}}\right\}<10^{-5}$ and the CPU time cost on single processor. The mass flow rates from a finite difference solver that implements the CIS [37] are also included to indicate accuracy of the current DG results. At small Knudsen numbers, the GSIS can significantly reduce the iteration steps, therefore the CPU time cost by the GSIS is much less than that of the CIS, and the smaller the Knudsen number the more saving of CPU time by the GSIS. For example, the GSIS can be 330 times and 4 times more efficient than the CIS at $\tau=0.01$ and 0.125 , respectively. When the Knudsen number is large, the GSIS uses almost the same number of iterations as the CIS to obtain the steady-state solution, although the GSIS costs about $10 \%$ to $20 \%$ more CPU time than the CIS as the additional synthetic equations are solved (An estimation of the computational complexity is given in Appendix B). Since the total number of iterations are small, both schemes are efficient.

To further demonstrate the ability of GSIS with fast convergence to steady-state solution for flow at small Knudsen number, we plot in Figure 4 the profiles of flow velocity at different iteration steps as well as the history of decay of the error $\epsilon=\max \left\{\mathcal{R}_{U_{1}}, \mathcal{R}_{q_{t, 1}}, \mathcal{R}_{q_{r, 1}}\right\}$ from the CIS and the GSIS at $\tau=0.01$. Starting from the initial value of $U_{1}=0$, it is found that for the CIS the perturbation in flow velocity from the external temperature gradient first arises near the walls and the gas in the bulk region remains undisturbed at the beginning of the iteration, e.g. at the 100-th iteration step; as the iteration proceeds, the perturbation gradually penetrates into the bulk region, and the scheme needs 8158 steps to achieve $\epsilon<10^{-5}$. The situation is completely changed in the GSIS, now with the aid of the synthetic equations, within the whole domain the macroscopic velocity emerges even after 1 iteration and the velocity profile is very close to the final steady state at the 5th iteration. Therefore the GSIS converges extremely fast, where the error decays by nearly 3 orders of magnitude within 10 iterations with a proper choice of $\tau_{t h}$, see Figure 4(c); such a behavior is consistent with the analytical calculation of the convergence rate in Figure 2 where the maximum value of $g_{0}$ is around 0.5 .

\subsection{Thermal conduction between parallel plates and inside a square cavity}

The second problem is the steady thermal conduction. We first consider two parallel plates located at $x_{2}=0$ and $x_{2}=1$, where the lower plate has a temperature of $\Delta T$ and the upper one has a temperature of $-\Delta T$. To compare our results with the DSMC data in Ref. 23], we set $\Delta T=0.0476$ and the gas parameters as $d_{r}=2, \omega=0.5, \delta=1 / 1.2, Z=5$. The two free parameters $\omega_{0}$ and $\omega_{1}$ are determined to recover the following Prandtl number Pr used in the DSMC:

$$
\operatorname{Pr}=\frac{5+d_{r}}{5 Z}\left[\frac{3}{2 Z+1-\omega_{0}}+\frac{d_{r} / 5}{\delta Z+(1-\delta)\left(1-\omega_{1}\right)}\right]^{-1}=0.73
$$

Note that equipped with the variable-soft-sphere model and the Borgnakke \& Larsen collision model [11, the DSMC can recover the experimental values of shear viscosity, bulk viscosity as well as self-diffusion coefficient, but it has no mechanism dedicated to recovering the thermal conductivity and its components [12]. Actually, we may have arbitrary combination of $\omega_{0}$ and $\omega_{1}$ from Eq. (46); this gives us the freedom to adjust the 

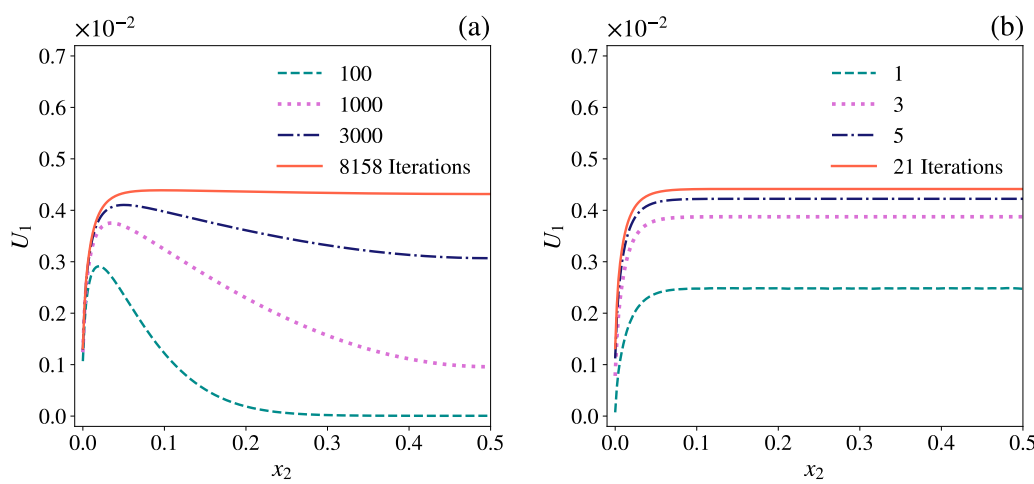

(b)

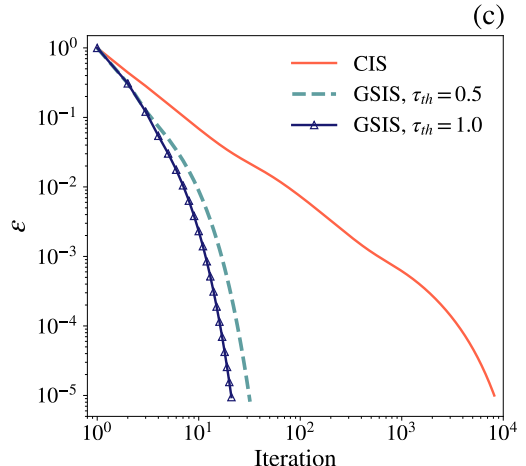

Figure 4: Profiles of flow velocity (only $U_{1}\left(0 \leq x_{2} \leq 0.5\right)$ is shown since the problem is symmetrical about $\left.x_{2}=0.5\right)$ for the thermal creep flow of nitrogen at different iteration steps obtained from: (a) CIS and (b) GSIS with $\tau_{t h}=1$. (c) The decay of the error $\epsilon=\max \left\{\mathcal{R}_{U_{1}}, \mathcal{R}_{q_{t, 1}}, \mathcal{R}_{q_{r, 1}}\right\}$ as a function of the iteration step with $\Delta t=\tau$. The Knudsen number is $\tau=0.01$. The Rykov model is solved with $d_{r}=2, \delta=1 / 1.33, Z=1.0, \omega_{0}=0.389$, and $\omega_{1}=1.787$.

Table 2: Comparison between GSIS and CIS for thermal conduction between two parallel plates in terms of the total heat flux $q\left(x_{2}=0.5\right)$ measured at the center of the domain, the number of iterations (Itr) to reach $\epsilon=\max \left\{\mathcal{R}_{\rho}, \mathcal{R}_{T_{t}}, \mathcal{R}_{T_{r}}\right\}<10^{-5}$ with $\Delta t=\tau / 4$ and $\tau_{t h}=0.5$, as well as the wall time cost on 4 processors using OpenMP for parallelism. The Boltzmann kinetic equations (3) and (4) are solved using $48 \times 48 \times 24$ discrete velocities over a truncated domain $[-6,6]^{3}$. The same number of frequencies are employed to calculate $\mathcal{L}_{\mathrm{BCO}}$ by the fast spectral method. The 4th-order DG scheme is applied on spatial mesh with 16 triangles. The gas properties are $\omega=0.5, d_{r}=2, \delta=1 / 1.2, Z=5.0, \omega_{0}=0.452$, and $\omega_{1}=1.797$. The results obtained from DSMC 23 is listed in the last column for reference.

\begin{tabular}{ccccccccc}
\hline \multirow{2}{*}{$\tau$} & \multicolumn{3}{c}{ GSIS } & & \multicolumn{3}{c}{ CIS } & \multirow{2}{*}{$q[23$} \\
\cline { 2 - 4 } \cline { 6 - 7 } & $q\left(x_{2}=0\right)$ & Itr & wall time $[\mathrm{s}]$ & & $q\left(x_{2}=0\right)$ & Itr & wall time $[\mathrm{s}]$ & \\
\hline 0.01 & 0.0022 & 61 & 98.5 & & - & - & - & 0.0023 \\
0.1 & 0.0173 & 56 & 89.7 & & 0.0167 & 479 & 676.3 & 0.0175 \\
1.0 & 0.0557 & 39 & 63.6 & & 0.0557 & 45 & 63.5 & 0.0557 \\
10.0 & 0.0761 & 64 & 103.5 & & 0.0761 & 64 & 90.3 & 0.0755 \\
\hline
\end{tabular}

translational Eucken factor independently, while other gas kinetic model does not have this capability. Such a translational Eucken factor, as will be shown later, affect the rarefied flow of molecular gas significantly. Here in order to make a definite calculation, we first determine $\omega_{0}$ from the first equation in Eq. (44) with given $\delta$ and $Z$, and then determine $\omega_{1}$ from Eq. (46); finally, we have $\omega_{0}=0.452$ and $\omega_{1}=1.797$.

The kinetic equations are solved by the GSIS and the CIS, respectively, where the time step for iteration is chosen as $\Delta t=\tau / 4$ to make the iteration stable. The truncated molecular velocity domain $[-6,6]^{3}$ is discretized by 48 non-uniform points described by Eq. (43) in $v_{1}$ and $v_{2}$ and 24 equidistant ones in $v_{3}$. The collision term $\mathcal{L}_{\mathrm{BCO}}$ is calculated by the fast spectral method using $48 \times 48 \times 24$ equidistant frequencies in the frequency domain, and 6-point Gauss-Legendre quadrature for integrations with respect to the solid angles in unit sphere 39. As shown in Figure 5(a), the two-dimensional spatial domain is partitioned by 16 triangles in the following way: first $N_{s}=9$ seeds with

$$
x_{2}=\left(10-15 s+6 s^{2}\right) s^{3}, \quad s=\frac{\left(0,1, \ldots, N_{s}-1\right)}{2\left(N_{s}-1\right)},
$$

are distributed along the $x_{2}$ direction to divide the domain into 8 rectangles; then each rectangle is split into 2 uniform triangles, which results in the maximum cell size (characterized by the height of triangle) being around 0.22 . The boundary conditions are the same as in the previous test case. The 4-th order DG method is used for the spatial discretization. In the GSIS, the threshold Knudsen number in Eq. (45) is set as $\tau_{t h}=0.5$.

We compare the density $\rho$ and translational temperature $T_{t}$ obtained from the GSIS with the DSMC 
(a)

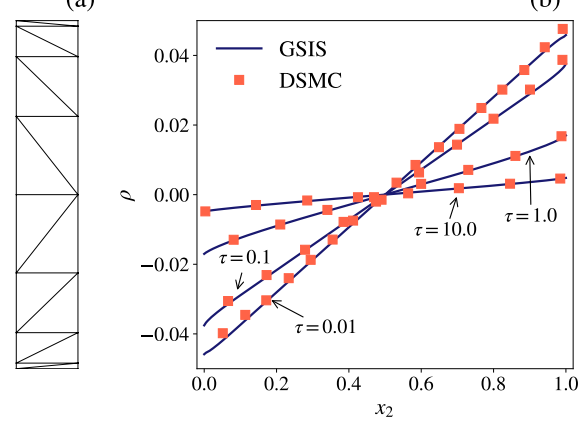

(b)

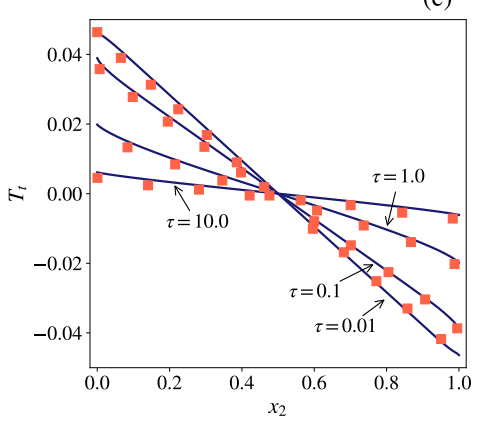

(c)

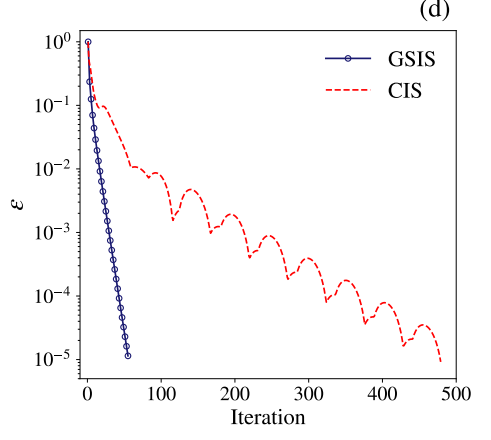

Figure 5: (a) Triangular mesh used to calculate the thermal conduction of molecular gas confined between two parallel plates. Comparisons between the GSIS and DSMC results [23] at $\tau=0.01,0.1,1$ and 10 on the (b) density $\rho$; and (c) translational temperature $T_{t}$. (d) Comparison between GSIS and CIS on the decay of the error $\epsilon=\max \left\{\mathcal{R}_{\rho}, \mathcal{R}_{T_{t}}, \mathcal{R}_{T_{r}}\right\}$ as a function of the iteration step with $\tau=0.1$ and $\Delta t=\tau / 4$. The Boltzmann kinetic equations (3) and (4) are solved with gas properties of $\omega=0.5, d_{r}=2, \delta=1 / 1.2, Z=5.0, \omega_{0}=0.452$, and $\omega_{1}=1.797$.

results at $\tau=0.01,0.1,1$ and 10 in Figure 5(b) and (c). The rotational temperature is very close to the translation temperature in this problem. Note that the DSMC data is obtained by solving the nonlinear version of Eqs. (3) 23. It is shown that the GSIS results are in good agreement with the DSMC data. The comparison between the GSIS and the CIS in terms of the total heat flux $q=q_{t, 2}+q_{r, 2}$ measured at $x_{2}=0.5$, the number of iteration to reach the criterion $\epsilon=\max \left\{\mathcal{R}_{\rho}, \mathcal{R}_{T_{t}}, \mathcal{R}_{T_{r}}\right\}<10^{-5}$, as well as the wall time cost on 4 processors using OpenMP for parallelism, are listed in Table 2 . The total heat flux obtained from the DSMC are also included. It is found that the GSIS costs about 40 to 60 iterations to find the steady state solutions for each case considered, and the maximum relative difference between $q$ from the GSIS and the one from DSMC is about $4 \%$. This further confirms the accuracy and efficiency of GSIS; especially, the GSIS can obtain accurate results on such coarse mesh at $\tau=0.01$, where the maximum cell size is about 22 times of the mean free path of gas molecules, due to the asymptotic preserving. Compared to the GSIS, the CIS requires almost the same number of iteration steps and slightly less computational time to obtain the same solutions at large Knudsen numbers, however, it needs many more iterations to reach the criterion of convergence than the GSIS at small Knudsen numbers. At $\tau=0.1$, the CIS needs 423 more iterations and nearly 7 times more computational time. The decays of the error $\epsilon$ for the GSIS and the CIS are shown in Figure 5(d), where it is found that the error in the CIS repeatedly oscillates before it is reduced to $10^{-5}$; this leads to the extremely slow convergence. Worse still, unlike the thermal creep flow in the previous section that it is well resolved by refined spatial mesh, the coarse mesh used here makes the CIS solutions contaminated by large numerical dissipation. For instance, the heat flux $q$ predicted by the CIS is smaller than the one from the GSIS at $\tau=0.1$; while it hardly obtains converged solutions at $\tau=0.01$, where the error $\epsilon$ is still larger than $10^{-2}$ even after 10000 iterations.

Now we push the simulation to the continuum limit to further show the asymptotic preserving property of GSIS, by considering the thermal conduction inside a two dimensional square cavity at $\tau=0.001$. The cavity is of dimensions $[0,1] \times[0,1]$, where the upper boundary is maintained at a higher temperature of $T_{H}=1$, and that of the other three walls is $T_{C}=0$. The analytical solution of temperature $T$ at the continuum limit is determined by the general heat equation $\nabla^{2} T=0$ :

$$
\frac{T-T_{C}}{T_{H}-T_{C}}=\frac{2}{\pi} \sum_{n=1}^{\infty} \frac{(-1)^{n+1}+1}{n} \sin \left(n \pi x_{1}\right) \frac{\sinh \left(n \pi x_{2}\right)}{\sinh (n \pi)} .
$$

We numerically solve this problem by the GSIS on the triangular mesh as illustrated in Figure 6(a). The mesh is generated in the following way: first 15 seeds are distributed along the $x_{1}$ and $x_{2}$ according to Eq. (47) to divide the domain into $14 \times 14$ rectangles; then each rectangle is partitioned into two uniform triangles. This results in a mesh with totally 392 triangles and the maximum (minimum) cell size is about 90 (2) times of the mean free path of gas molecules. The other computational configuration is the same as 
(a)

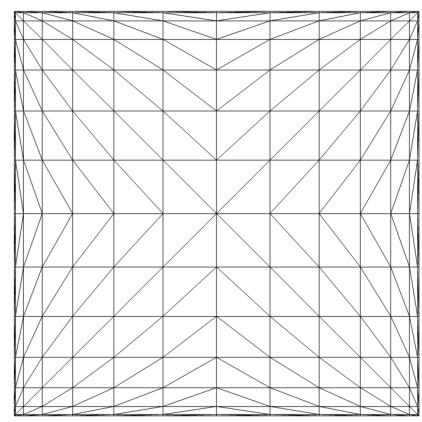

(b)

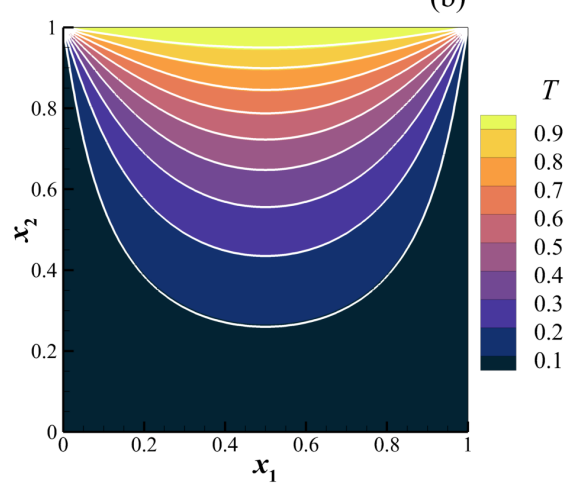

Figure 6: (a) Triangular mesh used to calculate the thermal conduction inside a square cavity. (b) Comparison on temperature $T$ obtained from GSIS (colored background) at $\tau=0.001$ and the analytical solution (white solid lines) of the steady heat equation at continuum limit. For GSIS, the Boltzmann kinetic equations (3) and 4 are solved with gas properties of $\omega=0.5$, $d_{r}=2, \delta=1 / 1.2, Z=5.0, \omega_{0}=0.452$, and $\omega_{1}=1.797$.

Table 3: The computational configurations to solve the Wu et al. for the thermal flow induced by temperature gradient of walls in a cavity: the number of triangles $N_{e l}$ for spatial discretization; the number of discrete molecular velocities $N_{v_{1}} \times$ $N_{v_{2}} \times N_{v_{3}}$; time step $\Delta t$ and threshold $\tau_{t h}$ for the iteration of GSIS. 'Itr' denotes the number of iterations to reach $\epsilon=$ $\max \left\{\mathcal{R}_{\rho}, \mathcal{R}_{T_{t}}, \mathcal{R}_{T_{r}}, \mathcal{R}_{|\boldsymbol{U}|}\right\}<10^{-5}$ and the wall time is that cost on 12 processors using OpenMP for parallelism. $32 \times 32 \times 24$ frequencies are employed to evaluate $\mathcal{L}_{\mathrm{BCO}}$. The locations of the center of the lower left vortex generated in the field are listed in the last column.

\begin{tabular}{cccccccc}
\hline$\tau$ & $N_{e l}$ & $N_{v_{1}} \times N_{v_{2}} \times N_{v_{3}}$ & $\Delta t$ & $\tau_{t h}$ & Itr & wall time $[\mathrm{s}]$ & $\left(x_{1}, x_{2}\right)$ \\
\hline 0.005 & 392 & $64 \times 64 \times 24$ & $\tau / 3$ & 0.5 & 49 & 375.5 & $(0.28,0.14)$ \\
0.05 & 288 & $64 \times 64 \times 24$ & $\tau / 3$ & 0.4 & 66 & 920.5 & $(0.27,0.17)$ \\
0.5 & 128 & $72 \times 72 \times 24$ & $\tau / 3$ & 0.5 & 73 & 1359.2 & $(0.24,0.18)$ \\
\hline
\end{tabular}

that for the one dimensional problem. Figure 6(b) plots the temperature contours obtained from the GSIS (terminated at the 69 th iteration when $\epsilon=\max \left\{\mathcal{R}_{\rho}, \mathcal{R}_{T_{t}}, \mathcal{R}_{T_{r}}\right\}<10^{-5}$ ) and the analytical solution (48). It is found that the two results are in good agreement. Therefore, the GSIS can recovery the Navier-Stokes solution without restricting the cell size comparable to or even smaller than the molecular mean free path.

\subsection{Thermal creep flow induced by temperature gradient of walls in a square cavity}

Now we consider more complicated flows that are seldom simulated by the DSMC or other deterministic solvers, that is a cavity flow induced by a temperature gradient at the wall from the early slip flow regime to the early transition regime, e.g. with the Knudsen numbers $\tau=0.005,0.05$ and 0.5. The computational domain is a $1 \times 1$ square, partitioned by the structured triangular mesh the same as shown in Figure 6(a). The boundaries are fully diffuse walls with the left and right ones maintained at a temperature $T_{C}$ and the other two having varied temperature given by

$$
T\left(x_{1}, x_{2}=0 \text { and } 1\right)= \begin{cases}2\left(T_{H}-T_{C}\right) x_{1}+T_{C}, & x_{1} \leq 0.5 \\ -2\left(T_{H}-T_{C}\right) x_{1}+2 T_{H}-T_{C}, & x_{1}>0.5\end{cases}
$$

where we set $T_{H / C}= \pm 0.03663$, respectively. The working gas is nitrogen of $\omega=0.74, d_{r}=2$ and $\delta=1 / 1.33$. The parameter $Z, \omega_{0}$ and $\omega_{1}$ are chosen to make the bulk viscosity and the thermal conductivity equal to the experimentally measured values. Note that it is convenient to use the Eucken factor to characterize the transfer of momentum and energy by molecules [4], which is defined as (in SI base unit)

$$
f_{e u}=\frac{m\left(\kappa_{t}+\kappa_{r}\right)}{\mu k_{\mathrm{B}}}=\frac{3}{2} f_{t}+\frac{d_{r}}{2} f_{r},
$$


(b)

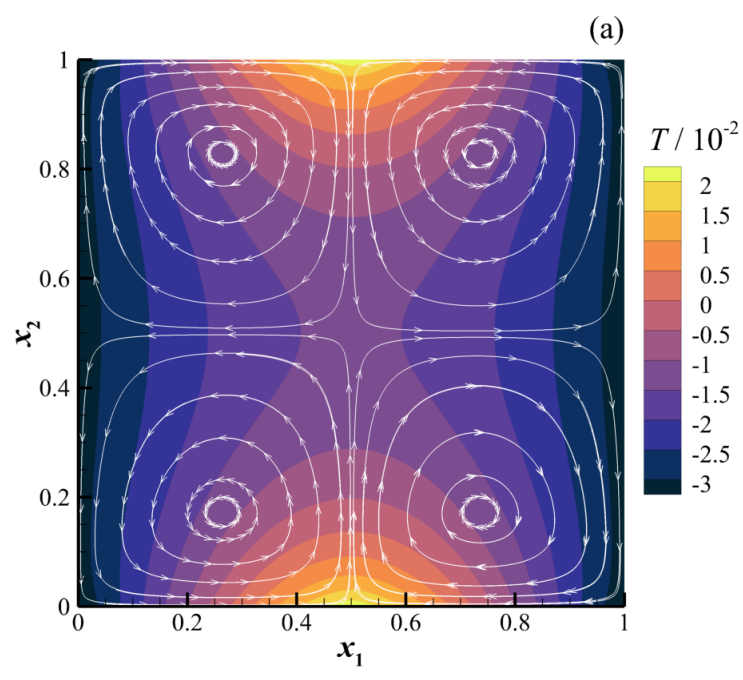

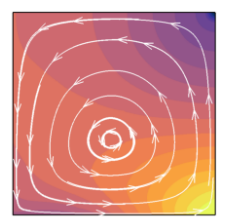
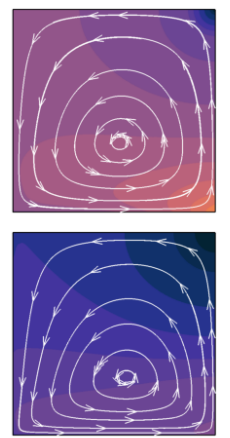

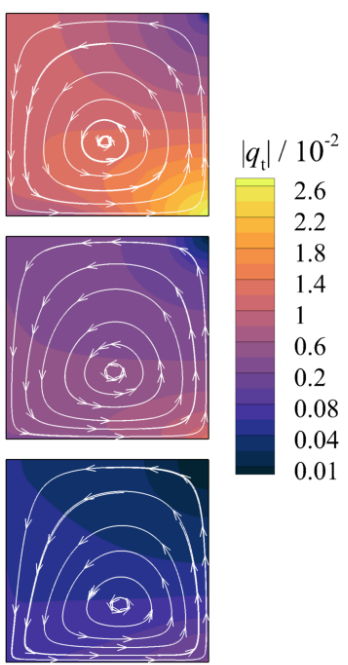

Figure 7: Thermal flow of nitrogen with $\omega=0.74, d_{r}=2, Z=2.235$ and $\delta=1 / 1.33$ induced by temperature gradient of walls in a cavity. (a) streamlines and background contours of the total temperature $T$ at Knudsen number $\tau=0.05$ with Eucken factors $f_{t}=2.24, f_{r}=1.54\left(\omega_{0}=0.481, \omega_{1}=1.924\right)$. (b) streamlines and background contours of the magnitude of translational heat flux $\left|\boldsymbol{q}_{t}\right|$ at different Knudsen numbers and translational Eucken factors: from the lower to the upper rows, $\tau=0.005,0.05$ and 0.5 , respectively; $f_{t}=2.24, f_{r}=1.54$ for the left column; $f_{t}=1.5, f_{r}=2.65\left(\omega_{0}=-1.98, \omega_{1}=4.374\right)$ for the right column. Only the lower left quarters of the flow fields are shown in (b).

with $f_{t}$ and $f_{r}$ being the translational and rotational Eucken factors, respectively. Therefore, from Eq. (5), we have

$$
\begin{aligned}
& f_{t}=\frac{5}{2}\left(1+\frac{1-\omega_{0}}{2 Z}\right)^{-1}, \\
& f_{r}=\left[\delta+\frac{\left(1-\omega_{1}\right)(1-\delta)}{Z}\right]^{-1} .
\end{aligned}
$$

Finally, the experiment of spontaneous Rayleigh-Brillouin scattering [12] shows that nitrogen has $Z=2.235$, $f_{t}=2.24$ and $f_{r}=1.54$ at the temperature of $273 \mathrm{~K}$, thus we have $\omega_{0}=0.481$ and $\omega_{1}=1.924$. For all the cases, the truncated molecular velocity domain is $[-6,6]^{3}$, which is discretized by $64 \times 64$ nonuniform points in $v_{1}$ and $v_{2}$ for $\tau=0.005$ and $\tau=0.05$, and $72 \times 72$ for $\tau=0.5$, while $v_{3}$ is discretized by 24 uniform points. To evaluate the Boltzmann collision operator, $32 \times 32 \times 24$ equidistant frequencies are employed. For spatial discretization, 392, 288 and 128 triangles are used for the cases of $\tau=0.005,0.05$ and 0.5 , respectively. The other parameters used in the GSIS as well as the number of iterations and computational time cost to obtain the steady-state solutions are listed in Table 3.

Figure 7(a) illustrates the counters of total temperature and streamlines for the flow at $\tau=0.05$. It is observed that higher temperature occurs near the centers of the lower and upper walls due to the heating from the walls, while lower temperature appears in the vicinity of four corners. The tangential temperature gradient along the lower and upper walls results in the thermal creep flows, where gas molecules moves from the cold regions to the hot ones. Eventually, 4 vortexes are generated within the domain with two rotating counter-clockwisely and the others rotating clockwisely. Note that the same flow for monatomic gas has been calculated by solving the Boltzmann equation in Ref. [41. The flow pattern of molecular gas is similar to that of the monatomic gas.

It has been found that, in thermal creep flow of molecular gas, the mass flow rate is proportional to the translation Eucken factor rather than the total one [45, 46, 47]. We also calculate the flows with different $f_{t}$ but the same $f_{e u}$. We plot the variations of the horizontal (vertical) velocities $U_{1}\left(U_{2}\right)$, the translational heat fluxes $q_{t, 1}\left(q_{t, 2}\right)$, as well as the rotational heat fluxes $q_{r, 1}\left(q_{r, 1}\right)$ along the vertical (horizontal) lines that cross 

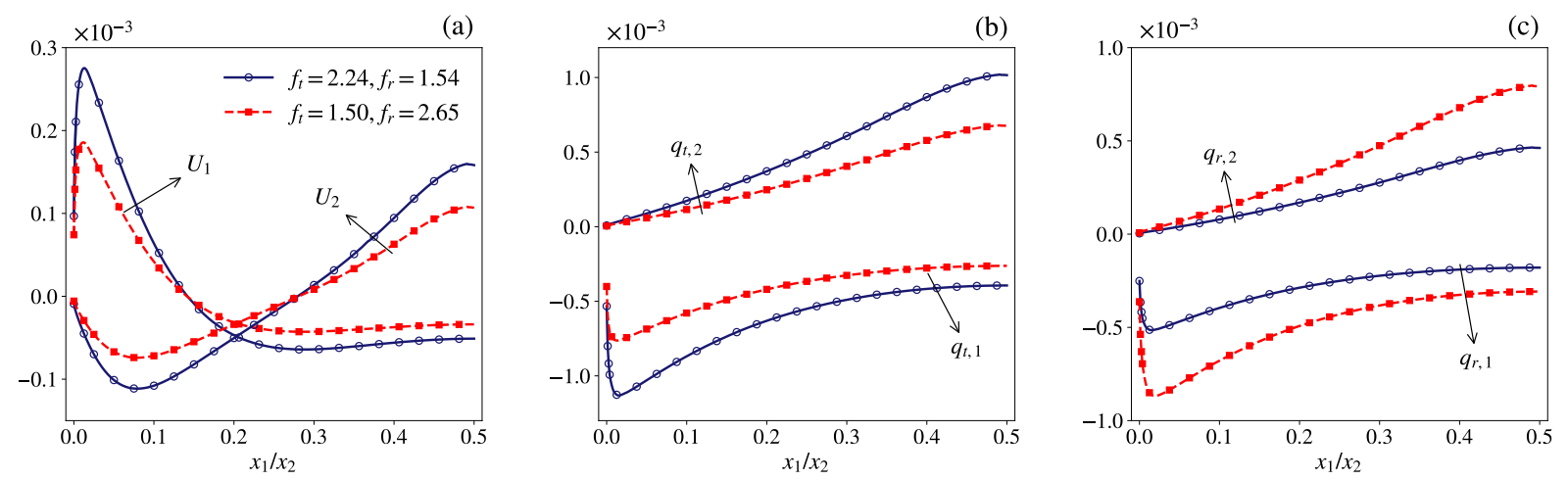

Figure 8: Thermal flow of nitrogen with $\omega=0.74, d_{r}=2, Z=2.235$ and $\delta=1 / 1.33$ induced by temperature gradient of walls in a cavity at $\tau=0.005$ : (a) the Horizontal/vertical velocities $U_{1} / U_{2}$, (b) the translational heat fluxes $q_{t, 1} / q_{t, 2}$ and (c) the rotational heat fluxes $q_{r, 1} / q_{r, 2}$ along the vertical/horizontal lines crossing the center of the lower left vortex (0.28, 0.14$)$. Only the lower left quarters of the flow fields are shown.

the center of the lower left vortex $(0.28,0.14)$ at $\tau=0.005$ in Figure 8 . The results are only shown within the lower left quarter of the computational domain due to symmetry of the flow field. It can be seen that with smaller $f_{t}$ but the same $f_{e u}$, the flow possesses smaller magnitude of bulk velocity and translational heat fluxes but larger rotational fluxes. The trends for flows at $\tau=0.05$ and 0.5 are similar. This further proves that the thermal creep flow is affected by the translational heat conduction. Figure 7(b) illustrate the counters of the magnitude of translational heat flux $\left|\boldsymbol{q}_{t}\right|$ and streamlines for $f_{t}=2.24$ and $f_{r}=1.54$ as well as those for $f_{t}=1.5$ and $f_{r}=2.65$, at different Knudsen numbers. It is observed that, at each Knudsen number, the heat transfer due to translational motion of molecules is strengthened near the center of the lower wall, however it becomes very weak in the center of the cavity. As the degree of rarefaction intensifies, the magnitude of heat flux generally increases. The center of the lower left vortex slightly moves towards the left upper domain with increasing Knudsen number, see the last column in Table 3 It is also shown that, smaller $f_{t}$ leads to smaller $\left|\boldsymbol{q}_{t}\right|$, however the location of the center of the vortex is not sensitive to the translational Eucken factor.

\subsection{Thermal driven flow induced by a hot beam in a rectangular chamber}

Finally, we simulate a two-dimensional thermal flow induced by a hot beam encompassed in a cold rectangular chamber, at $\tau=0.05,0.5$ and 5 . The beam, which is placed with a distance of 1 away from the left and bottom walls of the enclosure, has dimension of $4 \times 2$ and temperature of $T_{H}=1$, while the chamber has dimension of $10 \times 8$ and temperature of $T_{C}=0$, see Figure 9 (a). We consider a molecular gas that is filled between the beam and chamber. The gas parameters are set as: the viscosity index is $\omega=0.933$, the rotational degree of freedom is $d_{r}=2$, the Schmit number is $\delta=1 / 1.55$ and the rotational number is $Z=1.6$. $\omega_{0}=0.629$ and $\omega_{1}=0.519$ are obtained to have $f_{t}=2.24$ and $f_{r}=1.33$, respectively. Unlike the continuum flow where the flow velocity is zero and the temperature is governed by the Fourier's heat conduction law, at rarefied condition, the inhomogeneity of temperature induces not only energy exchange but also momentum transfer that in turn produces pressure gradient thus macroscopic flow velocity. This is a challenging problem for simulation, where the rarefaction effect should be well captured as it is the origin of the macroscopic motion. In addition, the magnitude of flow velocity is very small, e.g. it is at the order of $10^{-4}$ within most of the flow field when $\tau=0.05$. Therefore the numerical method should be accurate and efficient enough to well resolve the variation of flow velocity, meanwhile minimize numerical error to avoid contamination of the solution. We have shown that, insufficient resolution may lead to completely different and wrong result 35. Figure 9(b) illustrates the schematic of the spatial mesh, which is generated by structured triangles near the solid surfaces and unstructured triangles in the bulk region. Refinements are placed in the vicinity of the walls and near the corners of the beam for high resolutions of the Knudsen layer and singularity induced by the sharp edge. A total of 13960, 6630 and 2660 triangles are used for 
(a)

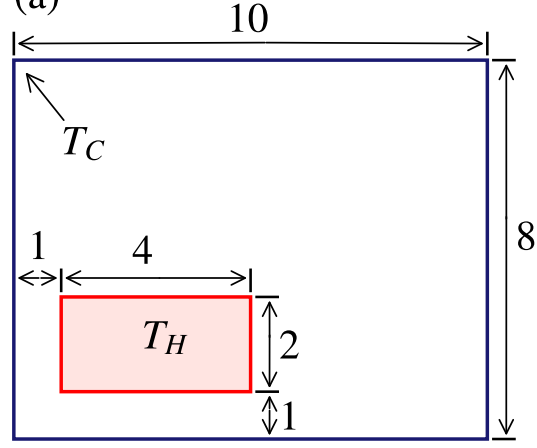

8 (b)

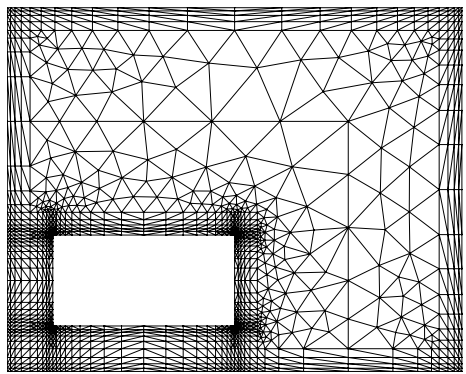

(c)

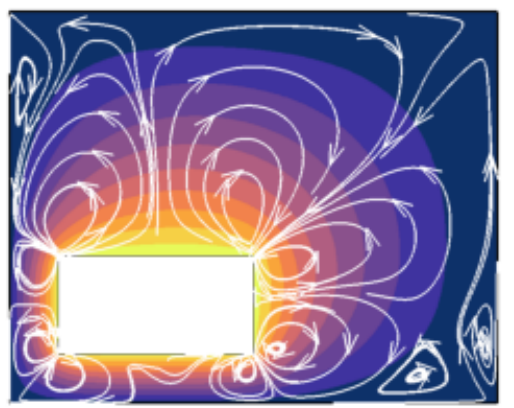

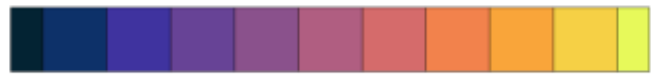

T: $\quad 0 \quad 0.10 .20 .30 .40 .50 .60 .70 .80 .9$

Figure 9: Thermal flow of a molecular gas with $\omega=0.933, d_{r}=2, Z=1.6, \delta=1 / 1.55, \omega_{0}=0.629$ and $\omega_{1}=0.519$ induced by a hot beam in a rectangular chamber. Schematic diagrams of (a) geometry and (b) spatial mesh. (c) From left to right: streamlines and background contours of total temperature $T$ at Knudsen number $\tau=0.05,0.5$ and 5 , respectively.
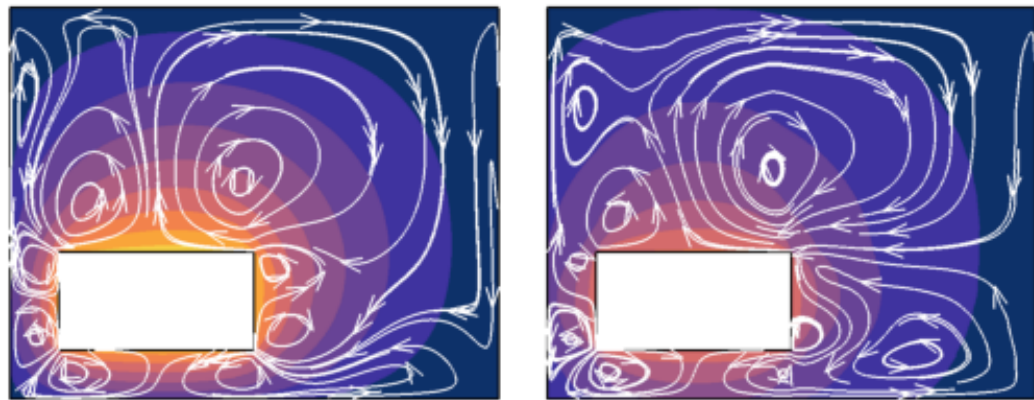

flows at $\tau=0.05,0.5$ and 5 , respectively. The minimum cell size is about $1 \%$ of the mean free path of gas molecules, while the maximum cell sizes are 75, 7.5 and 1.5 times of the mean free path when $\tau=0.05,0.5$ and 5, respectively. The 4th-order DG scheme is applied to solve the kinetic and synthetic equations. The truncated molecular velocity space $[-6,6]^{3}$ is discretized by $48 \times 48 \times 24$ nodes for $\tau=0.05$ and 0.5 , and by $96 \times 96 \times 24$ nodes for $\tau=5$, again, with nonuniform ones in $v_{1,2}$ and uniform ones in $v_{3}$. The configuration for the fast spectral method to evaluate $\mathcal{L}_{\mathrm{BCO}}$ is the same as that in the previous section. We set $\Delta t=\tau / 3$ and $\tau_{t h}=0.2 \sim 0.5$ to ensure the iteration of GSIS stable. Eventually, the GSIS cost $163(22.8 \mathrm{~h}), 109(7$ h) and $44(3.8 \mathrm{~h})$ iterations (the wall time on 28 processors) to obtained the steady-state solutions when $\epsilon=\max \left\{\mathcal{R}_{\rho}, \mathcal{R}_{T_{t}}, \mathcal{R}_{T_{r}}, \mathcal{R}_{|\boldsymbol{U}|}\right\}<10^{-5}$, for flows at $\tau=0.05,0.5$ and 5 , respectively. To the best knowledge of the authors, this is the most efficient solver for simulating such challenging problem.

The obtained contours of total temperature and streamlines are shown in Figure 9 (c). It is observed that the temperature of flow generally drops as the Knudsen number increases. When $\tau=0.05$, in the vicinity of each surface of the beam, the thermal flow drives gas molecules moving from the corners to the center of the surface, which forms a high pressure region therein and pushes the gas further into the void between the beam and chamber. Due to the confinement of the chamber walls, gas molecules finally return to the corners of the beam. As a consequence, eight vortices are generated around the corners of the beam. Besides, three more vortices can be observed near the upper-left and lower-right corners of the chamber. When $\tau$ increases to 0.5 and further to 5 , the two vortices near the lower-right corner of the chamber first merge as a larger vortice, which gradually grows up and swallows up the vortices close to it. Meanwhile, the vortex upon the upper-right corner of the beam also becomes larger and larger and swallows up the smaller vortices on its 
(a)

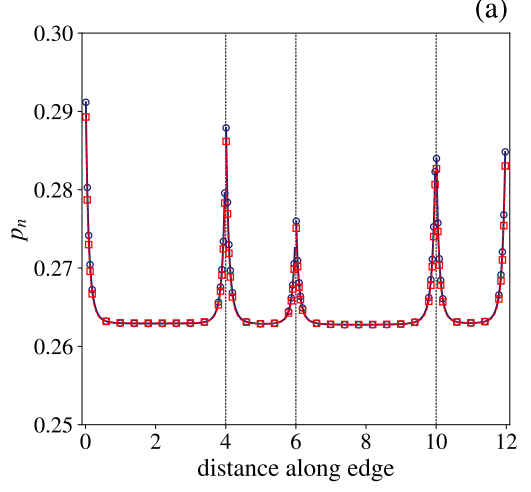

(d)

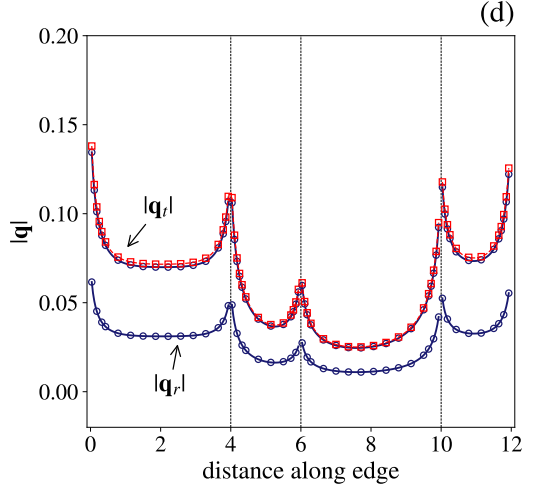

(b)

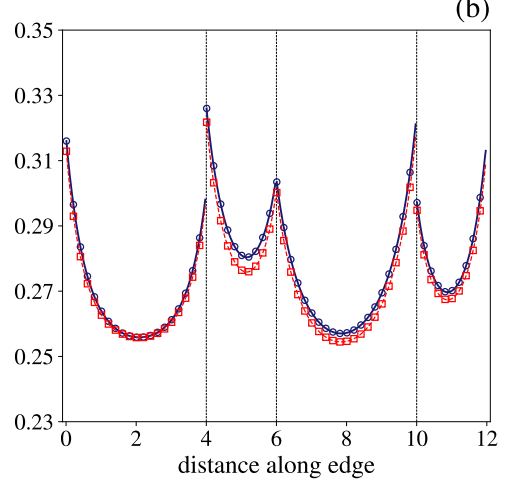

(e)

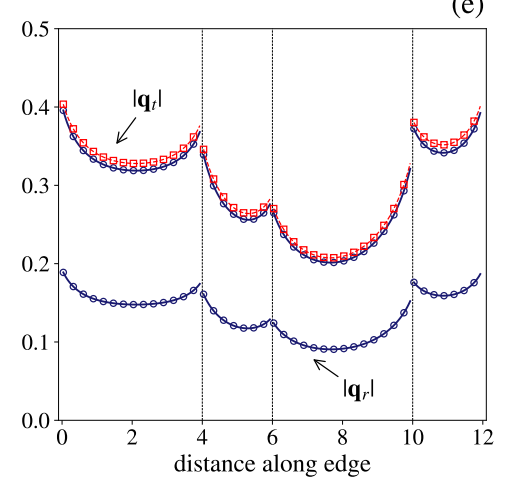

(c)

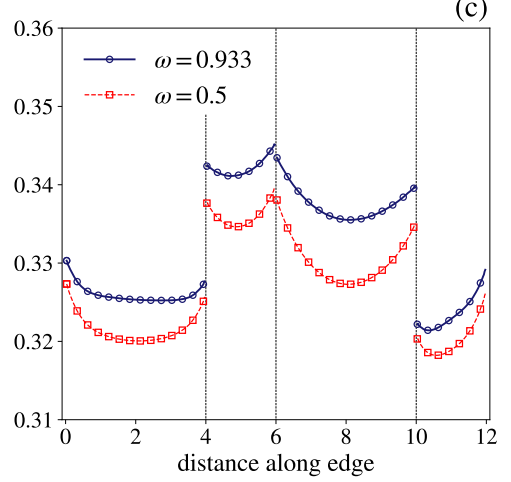

(f)

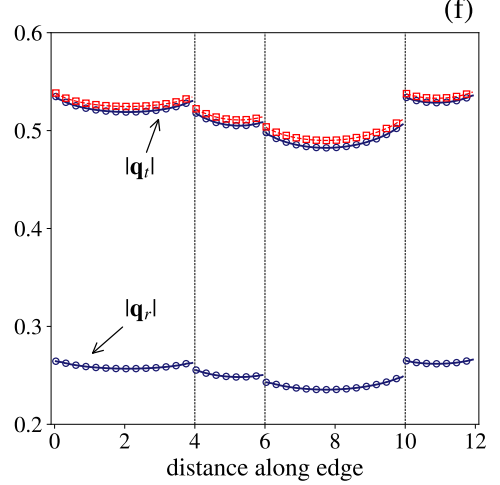

Figure 10: Thermal flow of a molecular gas with $\omega=0.933, d_{r}=2, Z=1.6, \delta=1 / 1.55, \omega_{0}=0.629$ and $\omega_{1}=0.519$ induced by a hot beam in a rectangular chamber. Normal stress $p_{n}$ (first row) and magnitude of heat fluxes $\left|\boldsymbol{q}_{t}\right|$ and $\left|\boldsymbol{q}_{r}\right|$ (second row) upon the surface of the hot beam, where the horizontal axis illustrates the distance along the edge from the lower left corner in a counter-clockwise manner. (a) and (d): $\tau=0.05$; (b) and (e): $\tau=0.5$; (c) and (f): $\tau=5$.

left side.

Figure 10 plots the normal stress $p_{n}$ and the magnitude of the translational (rotational) heat flux $\left|\boldsymbol{q}_{t}\right|$ $\left(\left|\boldsymbol{q}_{r}\right|\right)$ distributed on the surfaces of the hot beam. Here $p_{n}=\boldsymbol{n} \cdot \boldsymbol{P} \cdot \boldsymbol{n}$, with $\boldsymbol{n}$ the outward unit normal vector of the beam surface and $P_{i j}=2 \int v_{i} v_{j} h_{0} \mathrm{~d} \boldsymbol{v}$ the pressure tensor. It is observed that with increasing degree of rarefaction, i.e. fewer intermolecular collisions, the three quantities become larger and the difference between $\left|\boldsymbol{q}_{t}\right|$ and $\left|\boldsymbol{q}_{r}\right|$ intensifies. Furthermore, $p_{n}$ on each surface becomes more unbalanced, which mainly contributes to the arising of Knudsen force [48, 49. To include the effect of the shear viscosity index, we also calculate the flows with $\omega=0.5$ (now $\delta=1 / 1.2, \omega_{0}=0.629$ and $\omega_{1}=1.728$ to remain the same values of $f_{t}$ and $f_{r}$ ). It is found that smaller $\omega$ results in smaller $p_{n}$ but larger $\left|\boldsymbol{q}_{t}\right|$, and this effect is enhanced as the Knudsen number increases. The shear viscosity index has scarcely any influence on $\left|\boldsymbol{q}_{r}\right|$, thus we do not plot it for $\omega=0.5$ in Figure 10

\section{Conclusions}

We have proposed a general synthetic iterative scheme for rarefied polyatomic gas flows, with the aim to tackle the difficulties of the conventional iterative scheme, when solving the kinetic equation for gas flows in the near continuum flow regime. The key ingredient of GSIS is that a set of macroscopic equations are simultaneously solved with the gas kinetic equations, from which the flow density, the bulk velocity as well as the translational and rotational temperatures are obtained to guide the evolution of molecular velocity distribution functions. Due to the fact that the constitutive equations for the stress tensor and heat fluxes explicitly contain not only the Navier-stokes relations but also the high-order terms exactly derived from 
the kinetic equations, the proposed GSIS can achieve fast convergence to steady-state solutions in smallKnudsen-number flow regimes while produces results accuracy when the effect of rarefaction intensifies.

A rigorous Fourier stability analysis has been conducted to show that the maximum value of convergence rate for the extended GSIS can be less than 0.5, provided that a relaxation parameter (actually with quite large parameter window) for the correction of the velocity distribution functions from macroscopic quantities is properly chosen. This means that the scheme can reduce the difference of solutions between two successive iterative steps by at least three orders of magnitude only after 10 iterations, allowing fast convergence over the whole flow regime. Furthermore, through the Chapman-Enskog expansion, we have demonstrated that the macroscopic synthetic equations are reduced to the Navier-Stokes equations when the Knudsen number approaches zero, if the spatial cell size is adequate to resolve the hydrodynamic behaviors. The property of asymptotic preserving guarantees the GSIS to obtain accurate solution for small-Knudsen-number flows on the spatial cell size much larger than the mean free path of gas molecules.

The numerical results for the one-dimensional thermal creep flow and the thermal heat transfer between two parallel plates as well as the two-dimensional thermal heat transfer in a square cavity have shown that the proposed scheme can obtain the steady-state solutions within dozens of iterations when the Knudsen number varies from 0.001 to 10 . Meanwhile, the comparisons with the available DSMC data, the analytical solutions and the results from other deterministic solver prove accuracy of the GSIS. Especially, it can recover the solutions from continuum theory on a spatial mesh having maximum cell size of nearly 100 times larger than the molecular mean free path. The tests for the two-dimensional thermal creep flow and the thermal flow induced by the hot beam in the rectangular chamber have demonstrated the ability of the proposed GSIS to simulate more complicated flows and investigate the effects of the intermolecular potential (reflected in the viscosity index) and internal structure of gas molecules (reflected in the bulk viscosity, translational and rotational thermal conductivities) accurately and efficiently.

\section{Acknowledgments}

This work is supported in the UK by the Engineering and Physical Sciences Research Council under grant EP/R041938/1.

\section{Data Availability}

The data that support the findings of this study are available from the corresponding author upon reasonable request.

\section{Appendix A: matrices in the calculation of convergence rate}

In Section 4.1, we have shown that the convergence rate of CIS can be found by computing the eigenvalues of a $9 \times 9$ matrix $\mathbf{C}$, see Eq. 28). Here, the detail of $\mathbf{C}$ is given below,

$$
\mathbf{C}=\left[\begin{array}{l}
\mathbf{C}_{6,9} \\
\mathbf{C}_{3,9}
\end{array}\right], \quad \mathbf{C}_{6,9}=\left[\mathbf{C}_{1}, \mathbf{C}_{2}\right], \quad \mathbf{C}_{3,9}=\left[\mathbf{C}_{3}, \mathbf{C}_{4}^{\top}, \mathbf{C}_{5}, \mathbf{C}_{6}\right]
$$

where $\mathbf{C}_{2}, \mathbf{C}_{3} \mathbf{C}_{5}$ are zero matrices with dimension of $2 \times 2,3 \times 3$ and $3 \times 2$, respectively, while

$$
\begin{array}{r}
\mathbf{C}_{1}=\int y_{00}\left[1,2 v_{1}, 2 v_{2}, y_{14}, y_{15}, y_{16}, y_{17}\right]^{\top} \phi_{0} \mathrm{~d} \boldsymbol{v}, \\
\mathbf{C}_{4}=\frac{3 d_{r}}{2 Z\left(d_{r}+3\right)} \int y_{00} \mathrm{~d} \boldsymbol{v}, \\
\mathbf{C}_{6}=\int y_{00}\left[y_{61}, y_{62}, y_{63}\right]^{\top} \phi_{2} \mathrm{~d} \boldsymbol{v},
\end{array}
$$


with

$$
\begin{array}{r}
y_{00}=\frac{F_{e q}(\boldsymbol{v})}{1+\imath \tau \boldsymbol{\theta} \cdot \boldsymbol{v}} \phi_{2}, \quad y_{14}=\left[1-\frac{d_{r}}{Z\left(d_{r}+3\right)}\right]\left(|\boldsymbol{v}|^{2}-\frac{3}{2}\right), \\
y_{17}=\frac{d_{r}}{Z\left(d_{r}+3\right)}\left(|\boldsymbol{v}|^{2}-\frac{3}{2}\right), \quad y_{15}=\frac{4}{15}\left[1+\frac{\omega_{0}-1}{Z}\right] v_{1}\left(|\boldsymbol{v}|^{2}-\frac{5}{2}\right), \\
y_{16}=\frac{4}{15}\left[1+\frac{\omega_{0}-1}{Z}\right] v_{2}\left(|\boldsymbol{v}|^{2}-\frac{5}{2}\right), \quad y_{61}=\left[\frac{d_{r}}{2}-\frac{3 d_{r}}{2 Z\left(d_{r}+3\right)}\right], \\
y_{62}=2 \frac{\left(Z+\omega_{1}-1\right)(1-\delta)}{Z} v_{1}, \quad y_{63}=2 \frac{\left(Z+\omega_{1}-1\right)(1-\delta)}{Z} v_{2} .
\end{array}
$$

In the calculation of convergence rate for the GSIS according to Eq. 330 , the $9 \times 9$ matrix $\mathbf{L}$ is:

$$
\mathbf{L}=\left[\begin{array}{ccccccccc}
0 & \imath \theta_{1} & \imath \theta_{2} & 0 & 0 & 0 & 0 & 0 & 0 \\
\imath \theta_{1} & \tau & 0 & \imath \theta_{1} & 0 & 0 & 0 & 0 & 0 \\
\imath \theta_{2} & 0 & \tau & \imath \theta_{2} & 0 & 0 & 0 & 0 & 0 \\
0 & 0 & 0 & \frac{3 d_{r}}{2\left(d_{r}+3\right)} & \imath \theta_{1} & \imath \theta_{2} & -\frac{3 d_{r}}{2\left(d_{r}+3\right)} & 0 & 0 \\
0 & 0 & 0 & -\frac{3 d_{r}}{2\left(d_{r}+3\right)} & 0 & 0 & \frac{3 d_{r}}{2\left(d_{r}+3\right)} & \imath \theta_{1} & \imath \theta_{2} \\
0 & 0 & 0 & \kappa_{t} \tau \imath \theta_{1} & 1 & 0 & 0 & 0 & 0 \\
0 & 0 & 0 & \kappa_{t} \tau \imath \theta_{2} & 0 & 1 & 0 & 0 & 0 \\
0 & 0 & 0 & 0 & 0 & 0 & \kappa_{r} \tau \imath \theta_{1} & 1 & 0 \\
0 & 0 & 0 & 0 & 0 & 0 & \kappa_{r} \tau \imath \theta_{2} & 0 & 1
\end{array}\right]
$$

and the $9 \times 9$ matrix $\mathbf{R}$ is

$$
\mathbf{R}=\left[0, \mathbf{R}_{1}, \mathbf{R}_{2}, 0,0, \mathbf{R}_{t 1}, \mathbf{R}_{t 2}, \mathbf{R}_{r 1}, \mathbf{R}_{r 2}\right]^{\top},
$$

where

$$
\begin{aligned}
\mathbf{R}_{1} & =\int y_{00} w_{1}\left[1,2 v_{1}, 2 v_{2}, y_{14}, y_{15}, y_{16}, y_{17}, 0,0\right]^{\top} \mathrm{d} \boldsymbol{v}, \\
\mathbf{R}_{2} & =\int y_{00} w_{2}\left[1,2 v_{1}, 2 v_{2}, y_{14}, y_{15}, y_{16}, y_{17}, 0,0\right]^{\top} \mathrm{d} \boldsymbol{v}, \\
\mathbf{R}_{t 1} & =\int y_{00} w_{t 1}\left[1,2 v_{1}, 2 v_{2}, y_{14}, y_{15}, y_{16}, y_{17}, 0,0\right]^{\top} \mathrm{d} \boldsymbol{v}, \\
\mathbf{R}_{t 2} & =\int y_{00} w_{t 2}\left[1,2 v_{1}, 2 v_{2}, y_{14}, y_{15}, y_{16}, y_{17}, 0,0\right]^{\top} \mathrm{d} \boldsymbol{v}, \\
\mathbf{R}_{r 1} & =\int y_{00} w_{r 1}\left[0,0,0, \frac{3 d_{r}}{2 Z\left(d_{r}+3\right)}, 0,0, y_{61}, y_{62}, y_{63}\right]^{\top} \mathrm{d} \boldsymbol{v}, \\
\mathbf{R}_{r 2} & =\int y_{00} w_{r 2}\left[0,0,0, \frac{3 d_{r}}{2 Z\left(d_{r}+3\right)}, 0,0, y_{61}, y_{62}, y_{63}\right]^{\top} \mathrm{d} \boldsymbol{v},
\end{aligned}
$$


with

$$
\begin{aligned}
& w_{1}=\tau v_{1}+\tau\left(\imath \theta_{1} v_{1}+\imath \theta_{2} v_{2}\right)\left[\frac{2}{3} \imath \theta_{1}\left(2 v_{1}^{2}-v_{2}^{2}-v_{3}^{2}\right)+2 i \theta_{2} v_{1} v_{2}\right], \\
& w_{2}=\tau v_{2}+\tau\left(\imath \theta_{1} v_{1}+\imath \theta_{2} v_{2}\right)\left[2 \imath \theta_{1} v_{1} v_{2}+\frac{2}{3} \imath \theta_{2}\left(2 v_{2}^{2}-v_{1}^{2}-v_{3}^{2}\right)\right], \\
& w_{t 1}=\kappa_{t} \tau \imath \theta_{1}\left(\frac{2}{3}|\boldsymbol{v}|^{2}-1\right)-\frac{4 \kappa_{t} \tau}{5}\left(\imath \theta_{1} v_{1}^{2}+\imath \theta_{2} v_{1} v_{2}\right)\left(|\boldsymbol{v}|^{2}-\frac{5}{2}\right), \\
& w_{t 2}=\kappa_{t} \tau \imath \theta_{2}\left(\frac{2}{3}|\boldsymbol{v}|^{2}-1\right)-\frac{4 \kappa_{t} \tau}{5}\left(\imath \theta_{1} v_{1} v_{2}+\imath \theta_{2} v_{2}^{2}\right)\left(|\boldsymbol{v}|^{2}-\frac{5}{2}\right), \\
& w_{r 1}=\frac{2 \imath \theta_{1}}{d_{r}} \kappa_{r} \tau-\frac{4}{d_{r}} \kappa_{r} \tau\left(\imath \theta_{1} v_{1}^{2}+\imath \theta_{2} v_{1} v_{2}\right), \\
& w_{r 2}=\frac{2 \imath \theta_{2}}{d_{r}} \kappa_{r} \tau-\frac{4}{d_{r}} \kappa_{r} \tau\left(\imath \theta_{1} v_{1} v_{2}+\imath \theta_{2} v_{2}^{2}\right) .
\end{aligned}
$$

\section{Appendix B: discontinuous Galerkin method and boundary conditions}

We use the discontinuous Galerkin (DG) method to discretize the governing equations on triangular mesh in the physical space. Let $\Omega \in \mathbb{R}^{2}$ be a computational domain with boundary $\partial \Omega$ in the $x_{1}-x_{2}$ plane, which is partitioned into $N_{e l}$ disjoint regular triangles $\Delta_{l}: \Omega=\cup_{l}^{N_{e l}}\left\{\Delta_{l}\right\}$. The boundaries of the triangles define a group of $N_{f c}$ faces $\Gamma_{c}: \Gamma=\cup_{l}^{N_{e l}}\left\{\partial \Delta_{l}\right\}=\cup_{c}^{N_{f c}}\left\{\Gamma_{c}\right\}$. For the solution of gas kinetic equations, approximations of the reduced velocity distribution functions are sought in the following piecewise finite element spaces

$$
\mathcal{V}=\left\{\varphi:\left.\varphi\right|_{\Delta_{r}} \in \mathcal{P}^{K}\left(\Delta_{r}\right), \forall \Delta_{r} \subset \Omega\right\}
$$

where $\mathcal{P}^{K}(D)$ denotes the space of $K$-th order polynomials on a domain $D$. We introduce the notations $(a, b)_{D}=\int_{D \in \mathbb{R}^{2}}(a \odot b) \mathrm{d} x_{1} \mathrm{~d} x_{2}$ and $\langle a, b\rangle_{D}=\int_{D \in \mathbb{R}^{1}}(a \odot b) \mathrm{d} \Gamma$, where $\odot$ can be either the dot $(\cdot)$ or tensor $(\otimes)$ product. The DG formulation for CIS to find the approximations of the velocity distribution functions within each $\Delta_{l}$ at each iteration step is

$$
\frac{1}{\Delta t}\left(\varphi, h_{s}\right)_{\Delta_{l}}+\langle\varphi, \hat{\boldsymbol{H}} \cdot \boldsymbol{n}\rangle_{\partial \Delta_{l}}-\left(\nabla \varphi, \boldsymbol{v} h_{s}\right)_{\Delta_{l}}=\left(\varphi, \mathcal{C}_{s}\right)_{\Delta_{l}}+\frac{1}{\Delta t}\left(\varphi, h_{s}\right)_{\Delta_{l}}, \quad s=0,2
$$

where $\boldsymbol{n}$ is the outward unit normal vector and $\hat{\boldsymbol{H}}$ is the numerical flux that depends on the solutions from both sides of $\partial \Delta_{l}$ since the approximations of $h_{s}$ are discontinuous there. We calculate the numerical flux using the first-order upwind principle as

$$
\hat{\boldsymbol{H}} \cdot \boldsymbol{n}=\frac{1}{2} \boldsymbol{v} \cdot \boldsymbol{n}\left(h_{s}+h_{s}^{\mathrm{ext}}\right)+\frac{1}{2}|\boldsymbol{v} \cdot \boldsymbol{n}|\left(h_{s}-h_{s}^{\mathrm{ext}}\right),
$$

with $h_{s}^{\text {ext }}$ being the distribution from a neighboring triangle that shares the boundary $\partial \Delta_{l}$ with $\Delta_{l}$. If $\partial \Delta_{l}$ is at the boundary of computational domain, i.e. $\partial \Delta_{l} \cap \partial \Omega \neq 0, h_{s}^{\text {ext }}$ is evaluated using the given boundary condition. Once $h_{s}^{\text {ext }}$ is known, $h_{s}$ in $\Delta_{l}$ can be obtained by solving the linear system (B.2). A sweeping technique is utilized to find $h_{s}$ in an element-by-element fashion for all triangles [41. We also refer to the DG formulation incorporated into the fast spectral method for evaluating $\left(\varphi, \mathcal{L}_{\mathrm{BCO}}\right)_{\Delta_{l}}$ in Ref. [41].

The synthetic macroscopic equations are solved by the hybridizable discontinuous Galerkin (HDG) method, where the steady-state governing equations are first written in the following mixed system

$$
\begin{aligned}
\mathcal{G}_{u}+\nabla \cdot\left[\mathcal{G}_{c}+\mathcal{G}_{d}\right] & =0, \\
\boldsymbol{P}-\tau \nabla \boldsymbol{U}+\boldsymbol{\Pi} & =0, \\
\boldsymbol{E}-\tau \nabla T_{t}+\boldsymbol{\Theta} & =0, \\
\boldsymbol{W}-\tau \nabla T_{r}+\boldsymbol{\Lambda} & =0,
\end{aligned}
$$


where

$$
\begin{aligned}
& \mathcal{G}_{u}=\frac{3 d_{r}}{2 Z \tau\left(3+d_{r}\right)}\left[\begin{array}{c}
0 \\
\mathbf{0} \\
T_{t}-T_{r} \\
-T_{t}+T_{r}
\end{array}\right], \quad \mathcal{G}_{\mathrm{c}}=\left[\begin{array}{c}
\boldsymbol{U} \\
p_{t} \boldsymbol{I} \\
\mathbf{0} \\
\mathbf{0}
\end{array}\right], \quad \mathcal{G}_{d}=\left[\begin{array}{c}
\mathbf{0} \\
-\left(\boldsymbol{P}+\boldsymbol{P}^{\top}-\frac{2}{3} \operatorname{tr}(\boldsymbol{P}) \boldsymbol{I}\right) \\
-\kappa_{t} \boldsymbol{E} \\
-\kappa_{r} \boldsymbol{W}
\end{array}\right], \\
& \boldsymbol{\Pi}=\left[\begin{array}{cc}
\operatorname{HoT}_{\sigma_{11}}+\frac{1}{2} \mathrm{HoT}_{\sigma_{22}} & \frac{1}{2} \mathrm{HoT}_{\sigma_{12}} \\
\frac{1}{2} \mathrm{HoT}_{\sigma_{12}} & \frac{1}{2} \operatorname{HoT}_{\sigma_{11}}+\mathrm{HoT}_{\sigma_{22}}
\end{array}\right] \text {, } \\
& \boldsymbol{\Theta}=\frac{1}{\kappa_{t}}\left[\begin{array}{l}
\operatorname{HoT}_{q_{t, 1}} \\
\operatorname{HoT}_{q_{t, 2}}
\end{array}\right], \quad \boldsymbol{\Lambda}=\frac{1}{\kappa_{r}}\left[\begin{array}{l}
\operatorname{HoT}_{q_{r, 1}} \\
\operatorname{HoT}_{q_{r, 2}}
\end{array}\right],
\end{aligned}
$$

with $p_{t}=\rho+T_{t}$ the gas pressure associated to the translational motion and $\boldsymbol{I}$ the identity matrix. The auxiliary variables $\boldsymbol{P}, \boldsymbol{E}$ and $\boldsymbol{W}$ are introduced to approximate the velocity gradient $\nabla \boldsymbol{U}$ and temperature gradients $\nabla T_{t}$ and $\nabla T_{r}$. Then the stress tensor and heat fluxes are evaluated as

$$
\sigma_{i j}=-\left(P_{i j}+P_{j i}-\frac{2}{3} P_{k k} \delta_{i j}\right), \quad q_{t, i}=-\kappa_{t} E_{i}, \quad q_{r, i}=-\kappa_{r} W_{i}
$$

For the HDG discretization, the flow quantities $\boldsymbol{M}=\left[p_{t}, \boldsymbol{U}, T_{t}, T_{r}\right]$ as well as the auxiliary variables $\boldsymbol{P}, \boldsymbol{E}$ and $\boldsymbol{W}$ are approximated within $\Delta_{l}$ in the finite element space $\mathcal{V}$; the traces of the flow quantities $\hat{\boldsymbol{M}}=\left[\hat{p}_{t}, \hat{\boldsymbol{U}}, \hat{T}_{t}, \hat{T}_{r}\right]$ are approximated on the faces $\Gamma$ in the following piecewise finite element space

$$
\mathcal{W}=\left\{\psi:\left.\psi\right|_{\Gamma_{c}} \in \mathcal{P}^{K}\left(\Gamma_{c}\right), \forall \Gamma_{c} \subset \Gamma\right\}
$$

Generally speaking, when moving from the interior of triangle $\Delta_{l}$ to its boundary $\partial \Delta_{l}$, the traces define the values of field variables on the boundary. In HDG, it is assumed that the traces are singled-valued on each face.

The HDG method solves the system in two steps: first, a global problem is set up to determine the traces $\hat{\boldsymbol{M}}$; then a local problem with $\hat{\boldsymbol{M}}$ as boundary condition on $\partial \Delta_{l}$ is solved element-by-element to obtain the solutions for $\boldsymbol{M}, \boldsymbol{P}, \boldsymbol{E}$ and $\boldsymbol{W}$. The local problem is to find $(\boldsymbol{M}, \boldsymbol{P}, \boldsymbol{E}, \boldsymbol{W}) \in[\mathcal{V}]^{5} \times[\mathcal{V}]^{4} \times[\mathcal{V}]^{2} \times[\mathcal{V}]^{2}$ such that

$$
\begin{aligned}
\left(\boldsymbol{q}, \mathcal{G}_{u}\right)_{\Delta_{l}}-\left(\nabla \boldsymbol{q}, \mathcal{G}_{c}+\mathcal{G}_{d}\right)_{\Delta_{l}}+\langle\boldsymbol{q}, \hat{\boldsymbol{F}} \cdot \boldsymbol{n}\rangle_{\partial \Delta_{l}} & =0, \\
(\boldsymbol{r}, \boldsymbol{P})_{\Delta_{l}}+\tau(\nabla \cdot \boldsymbol{r}, \boldsymbol{U})_{\Delta_{l}}-\tau\langle\boldsymbol{r} \cdot \boldsymbol{n}, \hat{\boldsymbol{U}}\rangle_{\partial \Delta_{l}}+(\boldsymbol{r}, \boldsymbol{\Pi})_{\Delta_{l}} & =0, \\
(\boldsymbol{t}, \boldsymbol{E})_{\Delta_{l}}+\tau\left(\nabla \cdot \boldsymbol{t}, T_{t}\right)_{\Delta_{l}}-\tau\left\langle\boldsymbol{t} \cdot \boldsymbol{n}, \hat{T}_{t}\right\rangle_{\partial \Delta_{l}}+(\boldsymbol{t}, \boldsymbol{\Theta})_{\Delta_{l}} & =0, \\
(\boldsymbol{t}, \boldsymbol{W})_{\Delta_{l}}+\tau\left(\nabla \cdot \boldsymbol{t}, T_{r}\right)_{\Delta_{l}}-\tau\left\langle\boldsymbol{t} \cdot \boldsymbol{n}, \hat{T}_{r}\right\rangle_{\partial \Delta_{l}}+(\boldsymbol{t}, \boldsymbol{\Lambda})_{\Delta_{l}} & =0,
\end{aligned}
$$

for all $(\boldsymbol{q}, \boldsymbol{r}, \boldsymbol{t}) \in[\mathcal{V}]^{5} \times[\mathcal{V}]^{4} \times[\mathcal{V}]^{2}$, where the numerical flux $\hat{\boldsymbol{F}}$ is defined as:

$$
\hat{\boldsymbol{F}} \cdot \boldsymbol{n}=\left[\begin{array}{c}
\boldsymbol{U} \\
\hat{p}_{t}-\left(\boldsymbol{P}+\boldsymbol{P}^{\top}-\frac{2}{3} \operatorname{tr}(\boldsymbol{P}) \boldsymbol{I}\right) \\
-\kappa_{t} \boldsymbol{E} \\
-\kappa_{r} \boldsymbol{W}
\end{array}\right] \cdot \boldsymbol{n}+\left[\begin{array}{cccc}
1 & & & \\
& 1 & & \\
& & \kappa_{t} & \\
& & & \kappa_{r}
\end{array}\right]+\left[\begin{array}{c}
p_{t}-\hat{p}_{t} \\
\boldsymbol{U}-\hat{\boldsymbol{U}} \\
T_{t}-\hat{T}_{t} \\
T_{r}-\hat{T}_{r}
\end{array}\right] .
$$

The global problem is set up by enforcing the continuity of the numerical flux over all the interior faces. It is stated as: find $\hat{M} \in[\mathcal{W}]^{4}$ such that

$$
\left\langle(\hat{\boldsymbol{F}} \cdot \boldsymbol{n})^{+}, \boldsymbol{w}\right\rangle_{\Gamma_{c}}+\left\langle(\hat{\boldsymbol{F}} \cdot \boldsymbol{n})^{-}, \boldsymbol{w}\right\rangle_{\Gamma_{c}}=0, \quad \text { on } \Gamma_{c} \in \Gamma \backslash \partial \Omega,
$$

for all $\boldsymbol{w} \in[\mathcal{W}]^{4}$. Here the superscripts \pm denote the numerical fluxes obtained from the triangles on both sides of the face. Note that the traces on boundary faces are calculated from given boundary values $\boldsymbol{M}_{\mathrm{BC}}$

$$
\left\langle\hat{\boldsymbol{M}}-\boldsymbol{M}_{\mathrm{BC}}, \boldsymbol{w}\right\rangle_{\Gamma_{c}}, \quad \text { on } \Gamma_{c} \in \Gamma \cap \partial \Omega .
$$


By assembling the local problem $(\overline{B .8})$ and global problem $(\overline{B .10})$ and $(\overline{B .11})$ over all the triangles and faces, we can obtain a matrix system of form

$$
\left[\begin{array}{ccccc}
A_{M} & A_{P} & A_{E} & A_{W} & A_{\hat{M}} \\
B_{M} & B_{P} & B_{E} & B_{W} & B_{\hat{M}} \\
O_{M} & O_{P} & O_{E} & O_{W} & O_{\hat{M}} \\
Q_{M} & Q_{P} & Q_{E} & Q_{W} & Q_{\hat{M}} \\
Y_{M} & Y_{P} & Y_{E} & Y_{W} & Y_{\hat{M}}
\end{array}\right]\left[\begin{array}{c}
\mathbb{M} \\
\mathbb{P} \\
\mathbb{E} \\
\mathbb{W} \\
\hat{\mathbb{M}}
\end{array}\right]=\left[\begin{array}{c}
S_{M} \\
S_{P} \\
S_{E} \\
S_{W} \\
S_{\hat{M}}
\end{array}\right]
$$

where $\mathbb{M}, \mathbb{P}, \mathbb{E}, \mathbb{W}$ and $\hat{\mathbb{M}}$ are the vectors of degrees of freedom of the flow properties $\boldsymbol{M}$, the auxiliary variables $\boldsymbol{P}, \boldsymbol{E}$ and $\boldsymbol{W}$, as well as the trace of the flow properties $\hat{\boldsymbol{M}}$, respectively. Note that the degrees of freedom for $\boldsymbol{M}, \boldsymbol{P}, \boldsymbol{E}$ and $\boldsymbol{W}$ are grouped together and ordered element-by-element, and the corresponding coefficient matrix $\left[A_{M}, A_{P}, A_{E}, A_{W} ; B_{M}, B_{P}, B_{E}, B_{W} ; O_{M}, O_{P}, O_{E}, O_{W} ; Q_{M}, Q_{P}, Q_{E}, Q_{W}\right]$ has block diagonal structure. Therefore, we can eliminate $\boldsymbol{M}, \boldsymbol{P}, \boldsymbol{E}$ and $\boldsymbol{W}$ to obtained a reduced linear system involving only $\hat{\mathbb{M}}$. Once $\hat{\mathbb{M}}$ is determined, $\boldsymbol{M}, \boldsymbol{P}, \boldsymbol{E}$ and $\boldsymbol{W}$ are reconstructed corresponding to the local problem (B.8) in an element-wise fashion, while the stress tensor and the heat flux are calculated according to Eq. (B.6).

Now, we formulate the boundary condition, i.e. specify $h_{s}^{\text {ext }}$ and $\boldsymbol{M}_{\mathrm{BC}}$ at $\partial \Delta_{l}$ when $\partial \Delta_{l} \cap \partial \Omega \neq 0$. In this work, the static wall of temperature $T_{w}$ with fully diffuse reflection and periodic boundary are used. For the diffuse wall,

$$
h_{0}^{\text {ext }}=-\frac{1}{2} T_{w}-2 \sqrt{\pi} \int_{\boldsymbol{v} \cdot \boldsymbol{n}<0} \boldsymbol{v} \cdot \boldsymbol{n} h_{0}\left(\Delta_{l}, \boldsymbol{v}\right) \mathrm{d} \boldsymbol{v}+T_{w}\left(|\boldsymbol{v}|^{2}-\frac{3}{2}\right), \quad h_{2}^{\text {ext }}=\frac{d_{r}}{2} T_{w},
$$

where $\boldsymbol{n}$ is the outward unit normal vector of the wall, while $\boldsymbol{M}_{\mathrm{BC}}$ are directly calculated from the approximated velocity distribution functions within $\Delta_{l}$, in order to ensure correct velocity slippage and temperature jump, that is

$$
\left[\rho, \boldsymbol{U}, T_{t}\right]_{\mathrm{BC}}=\int\left[1, \boldsymbol{v}, \frac{2}{3}|\boldsymbol{v}|^{2}-1\right] h_{0}\left(\Delta_{l}, \boldsymbol{v}\right) \mathrm{d} \boldsymbol{v}, \quad\left[T_{r}\right]_{\mathrm{BC}}=\frac{2}{d_{r}} \int h_{2}\left(\Delta_{l}, \boldsymbol{v}\right) \mathrm{d} \boldsymbol{v} .
$$

In the case that $\partial \Delta_{l}$ lies on the periodic boundary, it is treated as an interior face between $\Delta_{l}$ and its fictitious counterpart (an imaginary triangle) across this boundary.

Finally, we estimate the computation effort to solve the system based on DG discretization at each iteration step. To solve the kinetic equations, we need to solve $N_{v_{1}} \times N_{v_{2}} \times N_{v_{3}} \times N_{e l}$ linear systems of (B.2), each of which involves $2 N_{d o f}^{e l}$ equations with $N_{d o f}^{e l}=(K+1)(K+2) / 2$ the number of degrees of freedom for a field variable in a triangle, since the kinetic equations are first discretized by $N_{v_{1}} \times N_{v_{2}} \times N_{v_{3}}$ discrete velocities in the molecular space before the DG discretization on spatial mesh. To evaluate $\left(\varphi, \mathcal{L}_{\mathrm{BCO}}\right)_{\Delta}$ through fast spectral method equipped with the FFT-based convolution, additional computational cost of $O\left(N_{d o f}^{e l} N_{e l} N_{q u a}^{2} \bar{N}^{3} \log \bar{N}+\left(N_{d o f}^{e l}\right)^{2} N_{e l} \bar{N}^{3}\right)$ is needed [41, where $N_{\text {qua }}$ is the number of quadrature points for the integrations with respect to the solid angles in unit sphere and $\bar{N}^{3}$ is the total number of frequencies. To solve the synthetic equations using the HDG method, the global problem to find the approximations of $\hat{M}$ is to solve a linear system for about $5 N_{f c} N_{d o f}^{f c}$ unknowns with $N_{d o f}^{f c}=K+1$ the number of degrees of freedom for a trace variable on a face. Note that the global linear system is sparse of about $5 N_{f c}\left(5 N_{d o f}^{f c}\right)^{2} / 2$ non-zero entities [50, which is solved by the direct solver PARDISO [51]. For the local problem, to recover $\boldsymbol{M}$ from $\hat{M}$ requires a cost of $O\left(N_{e l}\left(5 N_{d o f}^{e l}\right)^{2}\left(15 N_{d o f}^{f c}-1\right)\right)$.

\section{References}

[1] O. Popova, Meteoroid ablatioin models Earth, Moon, and Planets 95 (2004) 303-319.

[2] J. C. Maxwell, On the dynamical theory of gases, Philosophical Transactions of the Royal Society of London 157 (1867) 49-88.

[3] L. Boltzmann, Weitere Studien über das Wärmegleichgewicht unter Gasmolekülen Vieweg+Teubner Verlag, Wiesbaden, 1970, pp. 115-225. 
[4] G. A. Bird, Molecular Gas Dynamics and the Direct Simulation of Gas Flows, Clarendon Press, 1994.

[5] W. Wagner, A convergence proof for bird's direct simulation Monte Carlo method for the Boltzmann equation Journal of Statistical Physics 66 (1992) 1011-1044.

[6] J. Fan, C. Shen, Statistical simulation of low-speed rarefied gas flows Journal of Computational Physics 167 (2) (2001) $393-412$

[7] L. L. Baker, N. G. Hadjiconstantinou, Variance-reduced Monte Carlo solutions of the Boltzmann equation for low-speed gas flows: A discontinuous Galerkin formulation International Journal for Numerical Methods in Fluids 58 (4) (2008) 381-402.

[8] J. M. Burt, I. D. Boyd, A low diffusion particle method for simulating compressible inviscid flows Journal of Computational Physics 227 (9) (2008) $4653-4670$.

[9] C. Shen, Direct Simulation Monte-Carlo (DSMC) Method, 1st Edition, Rarefied Gas Dynamics, Springer, Berlin, 2005.

[10] K. Koura, H. Matsumoto, Variable soft sphere molecular model for inverse-power-law or lennard-jones potential Physics of Fluids A: Fluid Dynamics 3 (10) (1991) 2459-2465.

[11] C. Borgnakke, P. S. Larsen, Statistical collision model for Monte Carlo simulation of polyatomic gas mixture Journal of Computational Physics 18 (4) (1975) $405-420$.

[12] L. Wu, Q. Li, H. Liu, W. Ubachs, Extraction of the translational eucken factor from light scattering by molecular gas Journal of Fluid Mechanics 901 (2020) A23.

[13] S. Kosuge, K. Aoki, Shock-wave structure for a polyatomic gas with large bulk viscosity Physical Review Fluids 3 (2018) 023401 .

[14] L. Mieussens, Discrete-velocity models and numerical schemes for the Boltzmann-BGK equation in plane and axisymmetric geometries, Journal of Computational Physics 162 (2) (2000) $429-466$.

[15] J.-C. Huang, A conservative discrete ordinate method for model Boltzmann equations Computers \& Fluids 45 (1) (2011) 261 - 267, 22nd International Conference on Parallel Computational Fluid Dynamics (ParCFD 2010).

[16] S. Jaiswal, A. A. Alexeenko, J. Hu, A discontinuous Galerkin fast spectral method for the multi-species Boltzmann equation, Computer Methods in Applied Mechanics and Engineering 352 (2019) 56 - 84.

[17] C. S. Wang-Chang, G. E. Uhlenbeck, Transport phenomena in polyatomic gases, No. CM-681 Tech. rep. (1951).

[18] T. F. Morse, Kinetic model for gases with internal degrees of freedom The Physics of Fluids 7 (2) (1964) $159-169$.

[19] L. H. Holway, New statistical models for kinetic theory: Methods of construction. The Physics of Fluids 9 (9) (1966) 1658-1673.

[20] V. A. Rykov, A model kinetic equation for a gas with rotational degrees of freedom Fluid Dynamics (1975) 959-966.

[21] M. H. Gorji, P. Jenny, A fokker-planck based kinetic model for diatomic rarefied gas flows Physics of Fluids 25 (6) (2013) 062002 .

[22] V. A. Titarev, A. A. Frolova, Application of model kinetic equations to calculations of super- and hypersonic molecular gas flows, Fluid Dynamics 53 (2018) 536-551.

[23] C. Tantos, D. Valougeorgis, A. Frezzotti, Conductive heat transfer in rarefied polyatomic gases confined between parallel plates via various kinetic models and the DSMC method International Journal of Heat and Mass Transfer 88 (2015) 636 -651 .

[24] P. Wang, M. T. Ho, L. Wu, Z. Guo, Y. Zhang, A comparative study of discrete velocity methods for low-speed rarefied gas flows Computers \& Fluids 161 (2018) $33-46$.

[25] L. Wu, J. Zhang, H. Liu, Y. Zhang, J. M. Reese, A fast iterative scheme for the linearized Boltzmann equation Journal of Computational Physics 338 (2017) $431-451$.

[26] W. Su, P. Wang, Y. Zhang, L. Wu, A high-order hybridizable discontinuous Galerkin method with fast convergence to steady-state solutions of the gas kinetic equation Journal of Computational Physics 376 (2019) 973 - 991.

[27] M. L. Adams, E. W. Larsen, Fast iterative methods for discrete-ordinates particle transport calculations Progress in Nuclear Energy 40 (1) (2002) 3-159.

[28] C. Pain, M. Eaton, R. Smedley-Stevenson, A. Goddard, M. Piggott, C. de Oliveira, Space-time streamline upwind Petrov-Galerkin methods for the Boltzmann transport equation Computer Methods in Applied Mechanics and Engineering 195 (33) (2006) $4334-4357$.

[29] D. Valougeorgis, S. Naris, Acceleration schemes of the discrete velocity method: Gaseous flows in rectangular microchannels, SIAM Journal on Scientific Computing 25 (2) (2003) 534-552.

[30] S. Naris, D. Valougeorgis, D. Kalempa, F. Sharipov, Flow of gaseous mixtures through rectangular microchannels driven by pressure, temperature, and concentration gradients Physics of Fluids 17 (10) (2005) 100607.

[31] L. Szalmás, D. Valougeorgis, A fast iterative model for discrete velocity calculations on triangular grids Journal of Computational Physics 229 (11) (2010) $4315-4326$.

[32] W. Su, P. Wang, H. Liu, L. Wu, Accurate and efficient computation of the Boltzmann equation for couette flow: Influence of intermolecular potentials on Knudsen layer function and viscous slip coefficient Journal of Computational Physics 378 (2019) $573-590$.

[33] W. Su, L. Zhu, P. Wang, Y. Zhang, L. Wu, Can we find steady-state solutions to multiscale rarefied gas flows within dozens of iterations? Journal of Computational Physics 407 (2020) 109245.

[34] L. Zhu, X. Pi, W. Su, Z.-H. Li, Y. Zhang, L. Wu, General synthetic iteration scheme for non-linear gas kinetic simulation of multi-scale rarefied gas flows, arXiv (2020) 2004.10530

[35] W. Su, L. Zhu, L. Wu, Fast convergence and asymptotic preserving of the general synthetic iterative scheme, SIAM Journal on Scientific Computing (2020) Accepted.

[36] W. Su, M. T. Ho, Y. Zhang, L. Wu, GSIS: An efficient and accurate numerical method to obtain the apparent gas permeability of porous media Computers \& Fluids 206 (2020) 104576. 
[37] L. Wu, C. White, T. J. Scanlon, J. M. Reese, Y. Zhang, A kinetic model of the Boltzmann equation for non-vibrating polyatomic gases, Journal of Fluid Mechanics 763 (2015) 24-50.

[38] L. Wu, J. M. Reese, Y. Zhang, Solving the Boltzmann equation deterministically by the fast spectral method: application to gas microflows, Journal of Fluid Mechanics 746 (2014) 53-84.

[39] L. Wu, C. White, T. J. Scanlon, J. M. Reese, Y. Zhang, Deterministic numerical solutions of the Boltzmann equation using the fast spectral method. Journal of Computational Physics 250 (2013) $27-52$.

[40] S. Chapman, T. G. Cowling, The Mathematical Theory of Non-uniform Gases, Cambridge University Press, 1970.

[41] W. Su, P. Wang, Y. Zhang, L. Wu, Implicit Discontinuous Galerkin method for the Boltzmann equation, Journal of Scientific Computing 82 (2020) 39.

[42] E. M. Shakhov, Approximate kinetic equations in rarefied gas theory Fluid Dynamics 3 (1965) $112-115$.

[43] E. A. Mason, L. Monchick, Heat conductivity of polyatomic and polar gases The Journal of Chemical Physics 36 (6) (1962) 1622-1639.

[44] V. Lyusternik, R. Mustafaev, Eucken factor and the role of internal degrees of freedom in polyatomic hydrocarbon molecule transfer effects, Journal of Engineering Physics 31 (4) (1976) 1211-1214.

[45] S. K. Loyalka, T. S. Storvick, Kinetic theory of thermal transpiration and mechanocaloric effect. III. Flow of a polyatomic gas between parallel plates, The Journal of Chemical Physics 71 (1) (1979) 339-350.

[46] S. K. Loyalka, T. S. Storvick, S. S. Lo, Thermal transpiration and mechanocaloric effect. IV. Flow of a polyatomic gas in a cylindrical tube The Journal of Chemical Physics 76 (8) (1982) 4157-4170.

[47] P. Wang, W. Su, L. Wu, Thermal transpiration in molecular gas Physics of Fluids 32 (8) (2020) 082005.

[48] J. Nabeth, S. Chigullapalli, A. A. Alexeenko, Quantifying the Knudsen force on heated microbeams: A compact model and direct comparison with measurements Phys. Rev. E 83 (2011) 066306.

[49] Q. Li, T. Liang, W. Ye, Shape-dependent orientation of thermophoretic forces in microsystems Physical Review E 88 (2013) 033020 .

[50] A. Huerta, A. Angeloski, X. Roca, J. Peraire, Efficiency of high-order elements for continuous and discontinuous Galerkin methods, International Journal for Numerical Methods in Engineering 96 (9) (2013) 529-560.

[51] O. Schenk, K. Gärtner, Solving unsymmetric sparse systems of linear equations with PARDISO Future Generation Computer Systems 20 (3) (2004) 475 - 487, selected numerical algorithms. 